\title{
Variational description of bulk energies for bounded and unbounded spin systems
}

\author{
Roberto Alicandro, Marco Cicalese, Antoine Gloria
}

August 6, 2007

\begin{abstract}
We study the asymptotic behaviour of a general class of discrete energies defined on functions $u: \alpha \in \varepsilon \mathbb{Z}^{N} \cap \Omega \mapsto u(\alpha) \in \mathbb{R}^{m}$ of the form $E_{\varepsilon}(u)=\sum_{\alpha, \beta \in \varepsilon \mathbb{Z}^{N} \cap \Omega} \varepsilon^{N} g_{\varepsilon}(\alpha, \beta, u(\alpha), u(\beta))$, as the mesh size $\varepsilon$ goes to 0 . We prove that under general assumptions, that cover the case of bounded and unbounded spin systems in the thermodynamic limit, the variational limit of $E_{\varepsilon}$ has the form $E(u)=\int_{\Omega} g(x, u(x)) d x$. The cases of homogenization and of non-pairwise interacting systems (e.g. multiple-exchange spin-systems) are also discussed.
\end{abstract}

\section{Introduction}

Both in the applied mathematical and physical literature, there is much interest in the origin of pattern formation at the mesoscopic scale. On one side continuous descriptions provide a successful interpretation of pattern formation in terms of non attainment of infima (austenite/martensite phase transformations, micromagnetics in thin films, two wells problems etc., see $[5,21]$ and $[15,17,22,26]$ for reviews). On the other side, statistical mechanics aims at predicting such patterns starting from discrete systems of particles in interaction. The problem can be stated as follows. Given $m, L, N \in \mathbb{N}$ and $u: \mathbb{Z}^{N} \rightarrow \mathbb{R}^{m}$, an energy for a discrete system on $[0, L]^{N} \cap \mathbb{Z}^{N}$ in the configuration $u$ can be written as

$$
H_{L}(u)=\sum_{x \neq y \in \mathbb{Z}^{N} \cap[0, L]^{N}} g(x, y, u(x), u(y)) .
$$

According to the range of $u$ and the choice of $g$ (regarding the typical distance of the interactions, e.g.), we may recover many different models for spin systems, crystals, foams and polymers, to cite only a few of them. To study the macroscopic behaviour of such systems, one can characterize the thermodynamic limits of their free energies for general values of the temperature. In general, not much is known 
on the fine properties of the Gibbs states (such as pattern formation). At small temperature however, a good insight may consist in characterizing the ground states of the system at the bulk limit, namely:

$$
\lim _{L \rightarrow \infty} \frac{1}{L^{N}} \inf \left\{H_{L}(u), \text { boundary conditions }\right\} .
$$

There is actually a complete equivalence between letting the domain invade $\mathbb{R}^{N}$ (in the sense of Van Hove, e.g.) and taking the bulk limit on the one hand (as it is usually done in statistical mechanics [28]), and considering a fixed domain and letting the lattice spacing go to zero on the other hand. This point of view amounts to considering, for given $\Omega \subset \mathbb{R}^{N}$ and $\varepsilon>0$, the energy of a pairwise-interacting discrete system on $\mathbb{Z}_{\varepsilon}(\Omega):=\varepsilon \mathbb{Z}^{N} \cap \Omega$ in the configuration $u: \mathbb{Z}_{\varepsilon}(\Omega) \rightarrow \mathbb{R}^{m}$ with energy density $g_{\varepsilon}:\left(\mathbb{Z}_{\varepsilon}(\Omega)\right)^{2} \times \mathbb{R}^{2 m} \rightarrow \mathbb{R}$ on the lattice $\mathbb{Z}_{\varepsilon}(\Omega)$ as the family of functionals $E_{\varepsilon}: \mathbb{R}^{m} \rightarrow(-\infty,+\infty)$ defined as

$$
E_{\varepsilon}(u)=\sum_{\alpha, \beta \in \mathbb{Z}_{\varepsilon}(\Omega)} \varepsilon^{N} g_{\varepsilon}(\alpha, \beta, u(\alpha), u(\beta)) .
$$

By computing the $\Gamma$-limit of $E_{\varepsilon}$ as $\varepsilon$ goes to zero, the problem of getting some information on the ground states at the bulk limit can then be recast in terms of the study of fine properties of the minimizing sequences of the $\Gamma$-converging functionals $E_{\varepsilon}$. The latter is our point of view.

Within this setting, many authors have contributed to the study of the passage from discrete to continuum from a variational point of view for several interesting models in the framework of non-linear elasticity $([3,11,12])$, thin films elasticity ([1]), dislocations ([27]) and plasticity ([9]). Ising type energies for spin systems have also been studied recently in $[2,4]$, respectively for $u \in\{-1,+1\}$ and $u \in\left\{v \in \mathbb{R}^{m},|v|=1\right\}$. To compute the bulk limit for these systems is a trivial task, and fine properties of minimizers appear at a successive scale (interface or vortex-type phase transitions). This is not true in the general case. For instance, Giuliani, Lebowitz and Lieb [19] have recently addressed the characterization of ground states of a spin system mixing both short range ferromagnetic and long range antiferromagnetic interactions. For this model, the existence and the form of the bulk limit is not straightforward (see Section 6). Moreover the task of providing a finer analysis of the minimizers seems to be reasonably made easier if some information on the bulk limit is known. In particular, as the limit of a discrete system cannot always be written as a local integral functional (see [7]), the aim of the present paper is to find a wide class of energies of type (1.1) for which the $\Gamma$-limit can be written as

$$
E(u)=\int_{\Omega} g(x, u(x)) d x .
$$

Here we stress that the computation of this limit is the first necessary step, in the framework of expansion by $\Gamma$-convergence introduced by Braides and Truskinowsky in [13], towards the full analysis of a problem which entails multiple scales. 
To describe our results, it is useful to make a change of variables and rewrite the energies (1.1) as

$$
E_{\varepsilon}(u)=\sum_{\xi \in \mathbb{Z}^{N}} \sum_{\alpha, \alpha+\varepsilon \xi \in \mathbb{Z}_{\varepsilon}(\Omega)} \varepsilon^{N} f_{\varepsilon}^{\xi}(\alpha, u(\alpha), u(\alpha+\varepsilon \xi)) .
$$

In our analysis we distinguish whether the range of $u$ is bounded (or even a finite set) or not. The first case models classical spin systems, whereas the second one is usually referred to as the unbounded spin system case and has been first studied by Lebowitz and Presutti in [24] from the statistical mechanics point of view. We make two types of hypotheses on $f_{\varepsilon}^{\xi}$, namely growth conditions that ensure the limit functional to be finite on $L^{p}$ (for $1<p<\infty$ ) or on $L^{\infty}$, and a decay assumption on the range of the interactions that ensures the locality of the limit functional. Under this set of hypotheses we are able to prove a compactness theorem asserting that, up to a subsequence, $E_{\varepsilon} \Gamma$-converges to a functional of the form (1.2). To prove this result we use a well-known localization technique of the homogenization theory, which has been adapted to the discrete setting by two of the authors in [3]. It amounts to regarding the $\Gamma$-limit as a functional defined on pairs function-set and to proving that all the hypotheses of an integral representation result (see [14]) are satisfied.

We also study minimum problems with a constraint on the mean of the field $u$ (this constraint arises naturally in the context of spin systems). This analysis allows us to address the problem of homogenization for functionals of the type (1.1) when $f_{\varepsilon}^{\xi}(\cdot, u, v)=f^{\xi}(\dot{\varepsilon}, u, v)$ and $f^{\xi}(\cdot, u, v)$ is a periodic function. In particular in this case we prove that the $\Gamma$-limit is of the form

$$
\int_{\Omega} f_{h o m}(u(x)) d x
$$

and we provide a homogenization formula for the energy density

$$
f_{\text {hom }}(z)=\lim _{h \rightarrow+\infty} \frac{1}{h^{N}} \inf \left\{\mathcal{E}_{h}(u),\langle u\rangle=z\right\},
$$

where

$$
\mathcal{E}_{h}(u):=\sum_{\xi \in \mathbb{Z}^{N}} \sum_{\alpha, \alpha+\xi \in \mathbb{Z}^{N} \cap[0, h]^{N}} f^{\xi}(\alpha, u(\alpha), u(\alpha+\xi))
$$

and $\langle u\rangle=z$ stands for the mean of $u$ on $[0, h]^{N}$ (computed in a discrete sense). We then simplify the homogenization formula in the case of a density $f^{\xi}(\alpha, u, v)$ convex in the pair $(u, v)$.

In the last section of the paper we will see how these results can be extended to the case of more general spin systems driven by non-pairwise-interaction energies of the form

$$
F_{\varepsilon}(u)=\sum_{j=1}^{k} \sum_{\bar{\xi} \in \mathbb{Z}^{j N}} \sum_{\alpha, \alpha+\varepsilon \xi_{1}, \ldots, \alpha+\varepsilon \xi_{j} \in \mathbb{Z}_{\varepsilon}(\Omega)} \varepsilon_{\varepsilon}^{N} f_{\bar{\xi}}^{\bar{\xi}}\left(\alpha, u(\alpha), u\left(\alpha+\varepsilon \xi_{1}\right), \ldots, u\left(\alpha+\varepsilon \xi_{j}\right)\right)
$$


where $k \in \mathbb{N}$ and $\bar{\xi}=\left(\xi_{1}, \xi_{2}, \ldots, \xi_{j}\right) \in \mathbb{Z}^{j N}$. This class of discrete systems typically contains Heisenberg spin systems with multiple-spin exchange energies, whose energies are of the type

$$
F_{\varepsilon}(u)=\sum_{j=2}^{k} J_{j} \sum_{I^{j}} \varepsilon^{N} u\left(\alpha_{1}\right) u\left(\alpha_{2}\right) \ldots u\left(\alpha_{j}\right),
$$

where $k \geq 3, J_{j}$ are given constants, $K \in \mathbb{R}^{m}$ is a bounded set and $u \in K$. Here $I^{j}$ denotes a set of $j$-ples of points of the lattice subject to some geometric constraint. For this model we also provide, in Section 7.1, an example which gives us the opportunity to show how the limit energy density may depend on the geometric frustration of the spin system on different lattices.

As an example, in Section 6, we apply the result of the integral representation theorem to prove that the bulk limit of the ferromagnetic-antiferromagnetic model considered by Giuliani, Lebowitz and Lieb is a local integral.

The article is organized as follows:

\section{Contents}

1 Introduction $\quad 1$

2 Notation and preliminary results $\quad 5$

3 Compactness and integral representation results for spin systems 5

3.1 Pairwise-interaction energies . . . . . . . . . . . . . 6

3.2 Case $1<p<\infty$. . . . . . . . . . . . . . . . . . . . 7

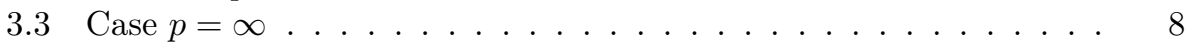

3.4 Proof in $L^{p}, 1<p<\infty \ldots \ldots \ldots$. . . . . . . . . . . . 10

3.5 Proof in $L^{\infty}$. . . . . . . . . . . . . . . . . . . . . . . . . . 16

4 Minimum problems $\quad 17$

5 Homogenization $\quad \mathbf{2 0}$

5.1 Homogenization in $L^{p}, 1<p<\infty$. . . . . . . . . . . . . . . 20

5.1 .1 The convex case ............... 23

5.2 Homogenization in $L^{\infty}$. . . . . . . . . . . . . . . . . . . . 25

6 Ferromagnetic-antiferromagnetic systems: existence of the bulk limit

7 Non-pairwise-interaction energies $\quad 29$

7.1 Multiple-spin exchange energies . . . . . . . . . . . . . . . 32 


\section{Notation and preliminary results}

In what follows $\mathcal{L}^{N}$ denotes the $N$-dimensional Lebesgue measure and $\Omega \subset \mathbb{R}^{N}$ is a bounded open set with $\mathcal{L}^{N}(\partial \Omega)=0$. Let $\mathcal{B}(\Omega)$ be the class of the Borel subsets of $\Omega, \mathcal{A}(\Omega)$ be the class of the open bounded subsets of $\Omega$, and $\mathcal{A}^{\mathcal{R}}(\Omega)$ be the class of the open bounded subsets $U \subset \Omega$ such that $\mathcal{L}^{N}(\partial U)=0$. For all $B \in \mathbb{R}^{N}$ we denote by $\mathbb{Z}_{\varepsilon}(B)=\varepsilon \mathbb{Z}^{N} \cap B$, and, for any $\xi \in \mathbb{Z}^{N}$, by $R_{\varepsilon}^{\xi}(B)=\left\{\alpha \in \varepsilon \mathbb{Z}^{N}: \alpha, \alpha+\varepsilon \xi \in B\right\}$. Given $k \in \mathbb{N}$ and $z \in \mathbb{R}^{m}$, we set $[z]_{k}:=k\left(\left[\frac{z_{1}}{k}\right],\left[\frac{z_{2}}{k}\right], \ldots,\left[\frac{z_{m}}{k}\right]\right)$.

We will also make use of the following integral representation theorem on Lebesgue spaces by Buttazzo and Dal Maso [14] for functionals defined on pairs function-sets:

Theorem 2.1 (Integral representation) Let $p \in\left[1, \infty\left[\right.\right.$, and let $F: L^{p}\left(\Omega, \mathbb{R}^{m}\right) \times$ $\mathcal{B}(\Omega) \rightarrow[0,+\infty]$ be a functional satisfying:

(i) $F$ is local on $\mathcal{B}(\Omega)$; i.e. $\forall u, v \in L^{p}\left(\Omega, \mathbb{R}^{m}\right)$ and $\forall B \in \mathcal{B}(\Omega), u=v$ a.e. on $B \Rightarrow F(u, B)=F(v, B)$;

(ii) $F$ is additive on $\mathcal{B}(\Omega)$; i.e. $\forall u \in L^{p}\left(\Omega, \mathbb{R}^{m}\right)$, and $\forall B_{1}, B_{2} \in \mathcal{B}(\Omega): B_{1} \cap B_{2}=$ $\emptyset \Rightarrow F\left(u, B_{1} \cup B_{2}\right)=F\left(u, B_{1}\right)+F\left(u, B_{2}\right) ;$

(iii) there exists $u_{0} \in L^{p}\left(\Omega, \mathbb{R}^{m}\right)$ such that $F\left(u_{0}, \cdot\right)$ is a Borel measure on $\mathcal{B}(\Omega)$ which is absolutely continuous w.r.t. $\mathcal{L}^{N}$,

(iv) the functional $F(\cdot, \Omega)$ is l.s.c. with respect to the weak convergence of $L^{p}\left(\Omega, \mathbb{R}^{m}\right)$,

then there exists a unique positive measurable function $f: \Omega \times \mathbb{R}^{m} \rightarrow[0,+\infty]$, with $f(x, \cdot)$ convex and lower semicontinuous for a.e. $x \in \Omega$, such that

$$
F(u, B)=\int_{B} f(x, u(x)) d x,
$$

for all $u \in L^{p}\left(\Omega, \mathbb{R}^{m}\right)$ and $B \in \mathcal{B}(\Omega)$.

If in addition there exist $D \in L^{1}\left(\Omega, \mathbb{R}^{m}\right), c, C>0$ such that

$$
c\|u\|_{L^{p}(B)}^{p} \leq F(u, B) \leq C\|u\|_{L^{p}(B)}^{p}+\|D\|_{L^{1}(B)}
$$

then $f$ is a Carathéodory function satisfying

$$
c|z|^{p} \leq f(x, z) \leq C|z|^{p}+D(x) \quad \text { for all } z \in \mathbb{R}^{m} \text { and } x \in \Omega
$$

\section{Compactness and integral representation results for spin systems}

In this section we define the class of energies we will mainly consider in the present work, i.e. pairwise-interaction energies. For this class of energies we prove a compactness and integral representation result asserting that, any sequence belonging 
to this family has a $\Gamma$-convergent subsequence whose $\Gamma$-limit is an integral functional.

Note that pairwise-interaction energies do not provide the most general setting to which our result apply. As it will be made precise in Section 7, Theorems 3.1 and 3.3 below can be extended to the case of systems driven by non-pairwise interaction energies. For the reader's convenience, we present the results for pairwiseinteraction energies, whose proofs contain all the ideas of the general case, and which allows us to avoid further technicalities and heavy notation.

\subsection{Pairwise-interaction energies}

Given $\Omega \subset \mathbb{R}^{N}$ and $\varepsilon>0$, the energy of a pairwise-interacting spin system with spin variable $u: \mathbb{Z}_{\varepsilon}(\Omega) \rightarrow \mathbb{R}^{m}$ and energy density $g_{\varepsilon}:\left(\mathbb{Z}_{\varepsilon}(\Omega)\right)^{2} \times \mathbb{R}^{2 m} \rightarrow \mathbb{R}$ on the lattice $\mathbb{Z}_{\varepsilon}(\Omega)$ is given by the functional $E_{\varepsilon}: \mathbb{R}^{m} \rightarrow(-\infty,+\infty)$ :

$$
E_{\varepsilon}(u)=\sum_{\alpha, \beta \in \mathbb{Z}_{\varepsilon}(\Omega)} \varepsilon^{N} g_{\varepsilon}(\alpha, \beta, u(\alpha), u(\beta)) .
$$

Observe that there is no loss of generality in considering the interactions symmetric. This symmetry condition is expressed by the formula $g_{\varepsilon}(\alpha, \beta, u, v)=$ $g_{\varepsilon}(\beta, \alpha, v, u)$ (note that, otherwise, one could deal with $\tilde{g}_{\varepsilon}(\alpha, \beta, u, v)=\frac{1}{2}\left(g_{\varepsilon}(\beta, \alpha, v, u)+\right.$ $\left.\left.g_{\varepsilon}(\alpha, \beta, u, v)\right)\right)$.

In the following we find it useful to rewrite the energy by a change of variable. Given $\xi \in \mathbb{Z}^{N}$ we define:

$$
g_{\varepsilon}(\alpha, \alpha+\varepsilon \xi, u, v)=f_{\varepsilon}^{\xi}(\alpha, u, v)
$$

and we have

$$
E_{\varepsilon}(u)=\sum_{\xi \in \mathbb{Z}^{N}} \sum_{\alpha \in R_{\varepsilon}^{\xi}(\Omega)} \varepsilon^{N} f_{\varepsilon}^{\xi}(\alpha, u(\alpha), u(\alpha+\varepsilon \xi)) .
$$

Note that, in the present variables, the symmetry condition reads $f_{\varepsilon}^{\xi}(\alpha, u, v)=$ $f_{\varepsilon}^{-\xi}(\alpha+\varepsilon \xi, v, u)$. Set, for any $k \in \mathbb{N}$,

$$
C_{\varepsilon}\left(\Omega, \mathbb{R}^{k}\right)=\left\{u: \mathbb{R}^{N} \rightarrow \mathbb{R}^{k}: u \text { constant on } \alpha+[0, \varepsilon)^{N} \text { for any } \alpha \in \mathbb{Z}_{\varepsilon}(\Omega)\right\} .
$$

We may identify any function $u: \mathbb{Z}_{\varepsilon}(\Omega) \rightarrow \mathbb{R}^{k}$ with a piecewise-constant function belonging to $C_{\varepsilon}\left(\Omega, \mathbb{R}^{k}\right)$ and then consider the family of energies $E_{\varepsilon}$ as defined on a subset of $L^{p}\left(\Omega, \mathbb{R}^{m}\right)$. Extending such energies on $L^{p}\left(\Omega, \mathbb{R}^{m}\right)$, we may define a family of functionals $F_{\varepsilon}: L^{p}\left(\Omega, \mathbb{R}^{m}\right) \rightarrow(-\infty,+\infty]$ by

$$
F_{\varepsilon}(u)= \begin{cases}\sum_{\xi \in \mathbb{Z}^{N}} \sum_{\alpha \in R_{\varepsilon}^{\xi}(\Omega)} \varepsilon^{N} f_{\varepsilon}^{\xi}(\alpha, u(\alpha), u(\alpha+\varepsilon \xi)) & \text { if } u \in C_{\varepsilon}\left(\Omega, \mathbb{R}^{m}\right) \\ +\infty & \text { otherwise, }\end{cases}
$$


where $f_{\varepsilon}^{\xi}: \mathbb{Z}_{\varepsilon}(\Omega) \times \mathbb{R}^{2 m} \rightarrow \mathbb{R}$ is a given function.

The set of hypotheses we are going to work with will depend on whether we consider the case $1<p<\infty$ or $p=\infty$.

\subsection{Case $1<p<\infty$}

Let us make the following hypotheses on the family of functions $f_{\varepsilon}^{\xi}$ :

(H1) Coercivity hypothesis. For all $\alpha, \xi$ and $\varepsilon$, there exist $c_{\varepsilon, \alpha}^{\xi} \geq 0$ and $d_{\varepsilon}^{\xi} \in$ $C_{\varepsilon}(\Omega, \mathbb{R}), d_{\varepsilon}^{\xi}(\alpha) \geq 0$ such that

$$
\begin{aligned}
& f_{\varepsilon}^{\xi}(\alpha, u, v) \geq c_{\varepsilon, \alpha}^{\xi}\left(|u|^{p}+|v|^{p}\right)-d_{\varepsilon}^{\xi}(\alpha) \quad \text { for all }(u, v) \in \mathbb{R}^{2 m} \\
& \lim _{R \rightarrow \infty} \liminf _{\varepsilon \rightarrow 0} \inf _{\alpha \in \mathbb{Z}_{\varepsilon}(\Omega)} \sum_{|\xi| \leq R} c_{\varepsilon, \alpha}^{\xi} \geq c>0
\end{aligned}
$$

and the function $d_{\varepsilon} \in C_{\varepsilon}(\Omega, \mathbb{R})$ defined by $d_{\varepsilon}(\alpha)=\sum_{\xi} d_{\varepsilon}^{\xi}(\alpha)$ weakly converges to $d$ in $L^{1}(\Omega)$.

(H2) Growth hypothesis. For all $\alpha, \xi$ and $\varepsilon$, there exist $C_{\varepsilon, \alpha}^{\xi} \geq 0$ and $D_{\varepsilon}^{\xi} \in$ $C_{\varepsilon}(\Omega, \mathbb{R}), D_{\varepsilon}^{\xi}(\alpha) \geq 0$ such that

$$
\begin{aligned}
& f_{\varepsilon}^{\xi}(\alpha, u, v) \leq C_{\varepsilon, \alpha}^{\xi}\left(|u|^{p}+|v|^{p}\right)+D_{\varepsilon}^{\xi}(\alpha) \text { for all }(u, v) \in \mathbb{R}^{2 m}, \\
& \limsup _{\varepsilon \rightarrow 0} \sup _{\alpha \in \mathbb{Z}_{\varepsilon}(\Omega)} \sum_{\xi \in \mathbb{Z}^{N}} C_{\varepsilon, \alpha}^{\xi} \leq C<\infty
\end{aligned}
$$

and the function $D_{\varepsilon} \in C_{\varepsilon}(\Omega, \mathbb{R})$ defined by $D_{\varepsilon}(\alpha)=\sum_{\xi} D_{\varepsilon}^{\xi}(\alpha)$ weakly converges to $D$ in $L^{1}(\Omega)$.

(H3) Decay hypothesis. For all $\delta>0$, there exists $M_{\delta}>0$ such that

$$
\limsup _{\varepsilon \rightarrow 0} \sup _{\alpha \in \mathbb{Z}_{\varepsilon}(\Omega)} \sum_{|\xi| \geq M_{\delta}} C_{\varepsilon, \alpha}^{\xi} \leq \delta .
$$

We will see that hypotheses $(\mathrm{H} 1)-(\mathrm{H} 2)$ ensure that any $\Gamma$-limit of a subsequence of $E_{\varepsilon}$ is defined on $L^{p}(\Omega)$. Hypothesis (H3) provides a control on the long-range interactions which yields the locality of the limit functional.

The main result of this section is the following

Theorem 3.1 Let $F_{\varepsilon}$ be as in (3.6), and $\left\{f_{\varepsilon}^{\xi}\right\}_{\varepsilon, \xi}$ satisfy hypotheses (H1), (H2) and (H3). Then, for every sequence converging to zero, there exists a subsequence 
$\left(\varepsilon_{j}\right)$ and a Carathéodory function $f: \Omega \times \mathbb{R}^{m} \rightarrow \mathbb{R}$ convex in the second variable and satisfying the following growth condition of order $p$

$$
c|y|^{p}-d(x) \leq f(x, y) \leq C|y|^{p}+D(x) \quad \text { for all } y \in \mathbb{R}^{m} \text { and } x \in \Omega,
$$

such that $\left(F_{\varepsilon_{j}}(\cdot)\right) \Gamma$-converges with respect to the weak convergence of $L^{p}\left(\Omega, \mathbb{R}^{m}\right)$ to the functional $F: L^{p}\left(\Omega, \mathbb{R}^{m}\right) \rightarrow \mathbb{R}$ defined by

$$
F(u)=\int_{\Omega} f(x, u(x)) d x .
$$

\subsection{Case $p=\infty$}

Let $K \subset \mathbb{R}^{m}$ be a bounded set. Let us make the following hypotheses on the family of functions $f_{\varepsilon}^{\xi}$ :

(H4) For all $\alpha, \xi$ and $\varepsilon, f_{\varepsilon}^{\xi}(\alpha, u, v)=+\infty$ if $(u, v) \notin K^{2}$,

(H5) For all $\alpha, \xi$ and $\varepsilon$, there exists $C_{\varepsilon, \alpha}^{\xi} \geq 0$ such that

$$
\begin{aligned}
& \left|f_{\varepsilon}^{\xi}(\alpha, u, v)\right| \leq C_{\varepsilon, \alpha}^{\xi} \quad \text { for all }(u, v) \in K^{2}, \\
& \limsup _{\varepsilon \rightarrow 0} \sup _{\alpha \in \mathbb{Z}_{\varepsilon}(\Omega)} \sum_{\xi \in \mathbb{Z}^{N}} C_{\varepsilon, \alpha}^{\xi}<\infty,
\end{aligned}
$$

(H6) for all $\delta>0$, there exists $M_{\delta}>0$ such that

$$
\limsup _{\varepsilon \rightarrow 0} \sup _{\alpha \in \mathbb{Z}_{\varepsilon}(\Omega)} \sum_{|\xi| \geq M_{\delta}} C_{\varepsilon, \alpha}^{\xi} \leq \delta .
$$

Remark 3.2 Hypotheses (H5) and (H6) do not imply $\limsup _{\varepsilon} \sum_{\xi} \sup _{\alpha} C_{\varepsilon, \alpha}^{\xi}<\infty$, as shown on the following example: $C_{\varepsilon, \alpha}^{\alpha / \varepsilon}=\frac{1}{\left|\frac{\alpha}{\varepsilon}\right|+1}$ and $C_{\varepsilon, \alpha}^{\xi}=0$ for $\xi \neq \frac{\alpha}{\varepsilon}$.

Theorem 3.3 Let $F_{\varepsilon}$ be as in (3.6), and $\left\{f_{\varepsilon}^{\xi}\right\}_{\varepsilon, \xi}$ satisfy hypotheses (H4), (H5) and (H6). Then, for every sequence converging to zero, there exists a subsequence $\left(\varepsilon_{j}\right)$ and a Carathéodory function $f: \Omega \times \bar{K} \rightarrow \mathbb{R}$ convex in the second variable such that $\left(F_{\varepsilon_{j}}(\cdot)\right) \Gamma$-converges with respect to the weak ${ }^{*}$-convergence of $L^{\infty}(\Omega, \mathbb{R})$ to the functional $F: L^{\infty}(\Omega, \mathbb{R}) \rightarrow \mathbb{R} \cup\{+\infty\}$ defined by

$$
F(u)= \begin{cases}\int_{\Omega} f(x, u(x)) d x & \text { if } u \in L^{\infty}(\Omega, \bar{K}) \\ +\infty & \text { otherwise, }\end{cases}
$$

where $\bar{K}$ is the convex hull of $K$ in $\mathbb{R}^{m}$. 
We now briefly discuss the optimality of hypothesis (H5) on two simple examples.

Example 3.4 In this example we show that if we weaken assumption (H5) by only assuming that

$$
\limsup _{\varepsilon}\left|\sum_{\xi} f_{\varepsilon}^{\xi}(\alpha, u, v)\right|<\infty \quad \forall \alpha \in R_{\varepsilon}^{\xi}(\Omega),(u, v) \in K^{2},
$$

then the $\Gamma$-limit may go to $-\infty$ at some point. Let us consider a one-dimensional discrete energy of the form (1.3) with an energy density given by:

$$
f_{\varepsilon}^{\xi}(\alpha, u, v)= \begin{cases}\frac{(-1)^{|\xi|+1}}{|\xi|+1} u v & \text { if } u, v \in\{-1,1\}, \\ +\infty & \text { if } u, v \notin\{-1,1\} .\end{cases}
$$

For $\Omega=[0,1]$ and $\varepsilon=\frac{1}{n}$, the energy of the system for $u: \frac{1}{n} \mathbb{Z} \cap[0,1] \rightarrow\{-1,1\}$ can be written as

$$
F_{n}(u)=\sum_{k=1}^{n} \frac{(-1)^{k+1}}{k+1} \sum_{i=0}^{n-k} \frac{1}{n} u\left(\frac{i}{n}\right) u\left(\frac{i+k}{n}\right) .
$$

Set $u_{n}\left(\frac{i}{n}\right)=(-1)^{i}$, we have $u_{n} \rightarrow^{*} 0$ in $L^{\infty}([0,1])$, and

$$
\lim _{n} F_{n}\left(u_{n}\right)=-\sum_{k=1}^{\infty} \frac{1}{k+1}=-\infty .
$$

Hence $\Gamma-\lim _{n} F_{n}(0)=-\infty$. However, $\Gamma-\lim _{n} F_{n}$ is not identically $-\infty$. Indeed it can be easily proved that

$$
\Gamma-\lim _{n} F_{n}(1)=\lim _{n} F_{n}(1)=\sum_{k=1}^{+\infty} \frac{(-1)^{k+1}}{k+1} .
$$

Example 3.5 In this example we weaken assumption (H5) by assuming that $C_{\varepsilon}^{e_{1}}$ goes to infinity as $\varepsilon \rightarrow 0$. Let us consider a one-dimensional nearest-neighbors spin system on $(0,1)$ with spin field taking values in $K=\{-1 ; 0 ; 1\}$. For $u: \varepsilon \mathbb{Z} \cap(0,1) \rightarrow$ $\{-1,0,1\}$, let the energy of the system be of the form

$$
F_{\varepsilon}(u)=\sum_{\alpha \in \varepsilon \mathbb{Z} \cap(0,1)} \varepsilon f_{\varepsilon}(u(\alpha), u(\alpha+\varepsilon)),
$$

where the pair potential $f_{\varepsilon}(u, v):\{-1 ; 0 ; 1\}^{2} \rightarrow(0,+\infty)$ is such that $f_{\varepsilon}(u, v)=$ $f_{\varepsilon}(v, u)$ and is given by

$$
f_{\varepsilon}(\alpha, u, v)= \begin{cases}\frac{1}{\varepsilon} & \text { if }(u, v)=(0,1) \\ 1 & \text { otherwise. }\end{cases}
$$


This energy does not satisfy (H5) since $f_{\varepsilon}(0,1) \rightarrow \infty$. However, any $u \in$ $L^{\infty}((0,1),[-1,1])$ can be approximated in the $w^{*}$-topology of $L^{\infty}$ by a sequence $u_{\varepsilon}: \varepsilon \mathbb{Z} \cap(0,1) \rightarrow\{-1,0,1\}$ such that $\left(u_{\varepsilon}(\alpha), u_{\varepsilon}(\alpha+\varepsilon)\right) \neq(0,1)$ for all $\alpha \in$ $\varepsilon \mathbb{Z} \cap(0,1)$. This suggests us that, if in the definition of $f_{\varepsilon}$ we replace $\frac{1}{\varepsilon}$ by any $C \geq \max \left\{f_{\varepsilon}(u, v),(u, v) \neq(0,1)\right\}$, then the modified energy satisfies assumption (H5) and has the same $\Gamma$-limit of the original one.

Let us turn to the case when in (3.10) the energy density (3.11) is replaced by

$$
f_{\varepsilon}(u, v)= \begin{cases}\frac{1}{\varepsilon} & \text { if }(u, v) \in\{(0,1),(-1,1)\} \\ \frac{1}{2} & \text { otherwise }\end{cases}
$$

and consider the piecewise constant function $u_{k}(x)=-1$ for $x<1 / k$, and $u_{k}(x)=$ 1 for $x \geq 1 / k$. For all $u_{\varepsilon} \rightarrow^{*} u_{k}$, we have $F_{\varepsilon}\left(u_{\varepsilon}\right) \geq 1+\frac{1}{2}+O(\varepsilon)=\frac{3}{2}+O(\varepsilon)$. This can be easily seen by minimizing pointwise the energy and noticing that we need at least one jump from 0 to 1 or from -1 to 1 to approximate $u_{k}$. Thus, if the $\Gamma-\lim _{\varepsilon} F_{\varepsilon}=: F$ exists, it satisfies $F\left(u_{k}\right) \geq \frac{3}{2}$. We also have $F(-1)=$ $F(1)=\frac{1}{2}$. Let us suppose now that $F$ admits an integral representation of the type $F(v)=\int_{0}^{1} f(x, v(x)) d x$. As $f \geq 0, F\left(u_{k}\right)=\int_{0}^{1 / k} f(x,-1)+\int_{1 / k}^{1} f(x, 1) \leq$ $\int_{0}^{1} f(x,-1)+\int_{0}^{1} f(x, 1)=F(-1)+F(1)=1$, which contradicts $F\left(u_{k}\right) \geq \frac{3}{2}$. Therefore the integral representation does not hold.

If $f_{\varepsilon}(0,1)=f_{\varepsilon}(-1,1)=\frac{1}{\varepsilon^{2}}$, we cannot even find sequences of equi-bounded energies converging to $u_{k}$. Therefore the $\Gamma$-limit is $+\infty$.

\subsection{Proof in $L^{p}, 1<p<\infty$}

In the proofs, we implicitly take $m=1$, since the arguments do not depend on the dimension (the problem is scalar as opposed to vectorial deal with in [3]).

To perform our analysis we need to define a localized version of the functional in (3.6). For any $A \in \mathcal{A}^{\mathcal{R}}(\Omega)$, we set for $F_{\varepsilon}(\cdot, A): L^{p}\left(\Omega, \mathbb{R}^{m}\right) \rightarrow(-\infty,+\infty]$

$$
F_{\varepsilon}(u, A)= \begin{cases}\sum_{\xi \in \mathbb{Z}^{N}} \sum_{\alpha \in R_{\varepsilon}^{\xi}(A)} \varepsilon^{N} f_{\varepsilon}^{\xi}(\alpha, u(\alpha), u(\alpha+\varepsilon \xi)) & \text { if } u \in C_{\varepsilon}\left(A, \mathbb{R}^{m}\right) \\ +\infty & \text { otherwise. }\end{cases}
$$


Moreover we define the lower and upper $\Gamma$-limits of $F_{\varepsilon}(\cdot, A)$ as

$$
\begin{aligned}
& F^{\prime}(u, A)=\Gamma-\liminf _{\varepsilon \rightarrow 0^{+}} F_{\varepsilon}(u, A)=\inf \left\{\liminf _{\varepsilon \rightarrow 0^{+}} F_{\varepsilon}\left(u_{\varepsilon}, A\right): u_{\varepsilon} \rightarrow u w-L^{p}(\Omega)\right\}, \\
& F^{\prime \prime}(u, A)=\Gamma-\limsup _{\varepsilon \rightarrow 0^{+}} F_{\varepsilon}(u, A)=\inf \left\{\limsup _{\varepsilon \rightarrow 0^{+}} F_{\varepsilon}\left(u_{\varepsilon}, A\right): u_{\varepsilon} \rightarrow u w-L^{p}(\Omega)\right\},
\end{aligned}
$$

respectively. Then the functional $F_{\varepsilon}$ is said to $\Gamma$-converge to $F$ as $\varepsilon \rightarrow 0^{+}$if and only if $F^{\prime}(u)=F^{\prime \prime}(u)=F(u)$ (we refer the reader to [8] and [16] for definitions and properties of $\Gamma$-convergence).

In the next two propositions we show that $(H 1)$ and (H2) imply that $F^{\prime}(u, A)$ and $F^{\prime \prime}(u, A)$ satisfy standard $p$-growth conditions.

Proposition 3.6 Let $A \in \mathcal{A}^{\mathcal{R}}(\Omega)$, and $\left\{f_{\varepsilon}^{\xi}\right\}$ satisfy (H1). If $u \in L^{p}(A)$ such that $F^{\prime}(u, A)<\infty$ then

$$
F^{\prime}(u, A) \geq c\left(\|u\|_{L^{p}(A)}^{p}-\|d\|_{L^{1}(A)}\right)
$$

for some positive constant $c$ independent of $u$ and $A$.

Proof. Let $\varepsilon_{n} \rightarrow 0$, and let $u_{n} \rightarrow u$ in $L^{p}(A)$ be such that $\liminf F_{\varepsilon_{n}}\left(u_{n}, A\right)<\infty$. We set $A_{\eta}=\{x \in A$ : $\operatorname{dist}(\mathrm{x}, \partial \mathrm{A})>\eta\}$ for all $\eta>0$. By the growth condition (H1), for $0<\eta^{\prime}<\eta$, we have

$$
\begin{aligned}
F_{\varepsilon_{n}}\left(u_{n}, A\right) & \geq \sum_{\alpha \in A_{\eta^{\prime}}} \sum_{|\xi| \leq \eta / \varepsilon_{n}} \varepsilon_{n}^{N} c_{\varepsilon_{n}, \alpha}^{\xi}\left|u_{n}(\alpha)\right|^{p}-\sum_{\xi \in \mathbb{Z}^{N}} \sum_{\alpha \in R_{\varepsilon_{n}}^{\xi}(A)} \varepsilon_{n}^{N} d_{\varepsilon_{n}}^{\xi}(\alpha) \\
& \geq \sum_{\alpha \in A_{\eta^{\prime}}} \varepsilon_{n}^{N} \inf _{\alpha \in \varepsilon_{n} \mathbb{Z}^{N} \cap \Omega}\left(\sum_{|\xi| \leq \eta / \varepsilon_{n}} c_{\varepsilon_{n}, \alpha}^{\xi}\right)\left|u_{n}(\alpha)\right|^{p}-\sum_{\xi \in \mathbb{Z}^{N}} \sum_{\alpha \in R_{\varepsilon_{n}}(A)} \varepsilon_{n}^{N} d_{\varepsilon_{n}}^{\xi}(\alpha) \\
& \geq \sum_{\alpha \in A_{\eta^{\prime}}} \varepsilon_{n}^{N} \frac{c}{2}\left|u_{n}(\alpha)\right|^{p}-\sum_{\xi \in \mathbb{Z}^{N}} \sum_{\alpha \in R_{\varepsilon_{n}}^{\xi}(A)} \varepsilon_{n}^{N} d_{\varepsilon_{n}}^{\xi}(\alpha) \\
& \geq \frac{c}{2} \int_{A_{\eta}}\left|u_{n}(x)\right|^{p} d x-\sum_{\xi \in \mathbb{Z}^{N}} \sum_{\alpha \in R_{\varepsilon_{n}}^{\xi}(A)} \varepsilon_{n}^{N} d_{\varepsilon_{n}}^{\xi}(\alpha)
\end{aligned}
$$

for $\varepsilon_{n}$ small enough. Using the lower semicontinuity of the norm for the weak convergence of $L^{p}$ and (H1), we obtain

$$
F^{\prime}(u, A) \geq \frac{c}{2} \int_{A_{\eta}}|u(x)|^{p} d x-\int_{A} d(x) d x
$$

Letting $\eta$ go to zero yields the thesis. 
Proposition 3.7 Let $A \in \mathcal{A}^{\mathcal{R}}(\Omega)$, and $\left\{f_{\varepsilon}^{\xi}\right\}$ satisfy (H2). If $u \in L^{p}(A)$ then

$$
F^{\prime \prime}(u, A) \leq C\left(\|u\|_{L^{p}(A)}^{p}+\|D\|_{L^{1}(A)}\right)
$$

for some positive constant $C$ independent of $u$ and $A$.

Proof. Let $u \in C^{0}(A)$ and let $u_{n}$ be defined by $u_{n}(\alpha)=u(\alpha)$ for all $\alpha$ such that $\alpha+[0, \varepsilon]^{N} \subset A$ and $u_{n}(\alpha)=0$ otherwise. We then have $u_{n} \rightarrow u$ in $L^{p}(A)$ and

$$
\begin{aligned}
F_{\varepsilon_{n}}\left(u_{n}, A\right) & \leq \sum_{\xi \in \mathbb{Z}^{N}} \sum_{\alpha \in R_{\varepsilon_{n}}^{\xi}(A)} \varepsilon_{n}^{N}\left(C_{\varepsilon_{n}, \alpha}^{\xi}\left(\left|u_{n}(\alpha)\right|^{p}+\left|u_{n}\left(\alpha+\varepsilon_{n} \xi\right)\right|^{p}\right)+D_{\varepsilon_{n}}^{\xi}(\alpha)\right) \\
& \leq 2 \sum_{\alpha \in \varepsilon_{n} \mathbb{Z}^{N} \cap A} \sum_{\xi \in \mathbb{Z}^{N}}^{N} C_{\varepsilon_{n}, \alpha}^{\xi}\left|u_{n}(\alpha)\right|^{p}+\sum_{\xi \in \mathbb{Z}^{N}} \sum_{\alpha \in R_{\varepsilon_{n}}^{\xi}(A)} \varepsilon_{n}^{N} D_{\varepsilon_{n}}^{\xi}(\alpha) \\
& \leq C \sum_{\alpha \in \varepsilon_{n} \mathbb{Z}^{N} \cap A} \varepsilon_{n}^{N}\left|u_{n}(\alpha)\right|^{p}+\sum_{\xi \in \mathbb{Z}^{N}} \sum_{\alpha \in R_{\varepsilon_{n}}^{\xi}(A)} \varepsilon_{n}^{N} D_{\varepsilon_{n}}^{\xi}(\alpha) \\
& \leq C \int_{A}\left|u_{n}(x)\right|^{p} d x+\sum_{\xi \in \mathbb{Z}^{N}} \sum_{\alpha \in R_{\varepsilon_{n}}^{\xi}(A)} \varepsilon_{n}^{N} D_{\varepsilon_{n}}^{\xi}(\alpha),
\end{aligned}
$$

due to the symmetry of the interactions. Letting $\varepsilon_{n}$ go to zero, we obtain

$$
F^{\prime \prime}(u, A) \leq C\left(\|u\|_{L^{p}(A)}^{p}+\|D\|_{L^{1}(A)}\right) .
$$

Using a density argument, we deduce the thesis for all $u \in L^{p}(A)$.

Remark 3.8 In order to prove Theorem 3.1 it is not restrictive to suppose that $f_{\varepsilon}^{\xi} \geq 0$. Indeed, if not, we can consider the family of functionals $\tilde{F}_{\varepsilon}(u, A)$ obtained by replacing $f_{\varepsilon}^{\xi}$ by $f_{\varepsilon}^{\xi}+d_{\varepsilon}^{\xi}$ in (3.12) and conclude using that $\tilde{F}^{\prime \prime}(u, A)=F^{\prime \prime}(u, A)+$ $\int_{A} d d x$.

As a consequence of the following three propositions we will prove that $F^{\prime \prime}(u, A)$ satisfy all the hypotheses of Theorem 2.1.

Proposition 3.9 Let $\left\{f_{\varepsilon}^{\xi}\right\}$ satisfy (H1)-(H3) and be such that $f_{\varepsilon}^{\xi} \geq 0$. If $u \in$ $L^{p}(\Omega)$ and $A \in \mathcal{A}^{\mathcal{R}}(\Omega)$, there holds

$$
\sup _{A^{\prime} \subset \subset A} F^{\prime \prime}\left(u, A^{\prime}\right)=F^{\prime \prime}(u, A) .
$$

Proof. By the non negativity of $f_{\varepsilon}^{\xi} \geq 0, F^{\prime \prime}(u, \cdot)$ is an increasing set function. Thus it suffices to prove

$$
\sup _{A^{\prime} \subset \subset A} F^{\prime \prime}\left(u, A^{\prime}\right) \geq F^{\prime \prime}(u, A)
$$


Given $\delta>0$, there exists $A^{\prime \prime} \subset \subset A$ such that

$$
\|D\|_{L^{1}\left(A \backslash \overline{A^{\prime \prime}}\right)}+\|u\|_{L^{p}\left(A \backslash \overline{A^{\prime \prime}}\right)}^{p} \leq \delta .
$$

Reasoning by approximation, we may find $v_{\varepsilon} \in L^{p}(\Omega)$ such that $v_{\varepsilon}$ weakly converges to $u$ in $L^{p}(\Omega)$ and

$$
\limsup _{\varepsilon \rightarrow 0} F_{\varepsilon}\left(v_{\varepsilon}, A \backslash \overline{A^{\prime \prime}}\right) \leq C\left(\|D\|_{L^{1}\left(A \backslash \overline{A^{\prime \prime}}\right)}+\|u\|_{L^{p}\left(A \backslash \overline{A^{\prime \prime}}\right)}^{p}\right) \leq C \delta .
$$

Let $A^{\prime} \in \mathcal{A}(\Omega)$ be such that $A^{\prime \prime} \subset \subset A^{\prime} \subset \subset A$ and let $u_{\varepsilon} \in L^{p}(\Omega)$ weakly converge to $u$ in $L^{p}(\Omega)$, with

$$
\limsup _{\varepsilon \rightarrow 0} F_{\varepsilon}\left(u_{\varepsilon}, A^{\prime}\right)=F^{\prime \prime}\left(u, A^{\prime}\right)
$$

Set

$$
d:=\operatorname{dist}\left(A^{\prime \prime}, A^{\prime c}\right)
$$

and for any $M \in \mathbb{N}$ and $i \in\{1, \ldots, M\}$ define

$$
A_{i}=\left\{x \in A: \operatorname{dist}\left(x, A^{\prime \prime}\right)<i \frac{d}{M}\right\} .
$$

Let $\varphi_{i}$ be the characteristic function of $A_{i}$. Then for any $i \in\{1, \ldots, M\}$ consider the family of functions $w_{\varepsilon}^{i} \in \mathcal{A}_{\varepsilon}(\Omega)$ still weakly converging to $u$ in $L^{p}(\Omega)$ defined by

$$
w_{\varepsilon}^{i}(\alpha):=\varphi_{i}(\alpha) u_{\varepsilon}(\alpha)+\left(1-\varphi_{i}(\alpha)\right) v_{\varepsilon}(\alpha) .
$$

Fix $i \in\{1,2, \ldots, M-3\}$. Given $\xi \in \mathbb{Z}^{N}$ and $\alpha \in R_{\varepsilon}^{\xi}(A)$, then either $\alpha \in$ $R_{\varepsilon}^{\xi}\left(A_{i}\right)$, or $\alpha \in R_{\varepsilon}^{\xi}\left(A \backslash \bar{A}_{i+1}\right)$, or

$$
[\alpha, \alpha+\varepsilon \xi] \cap\left(\bar{A}_{i+1} \backslash A_{i}\right) \cap{\overline{A^{\prime}}}^{c} \neq \emptyset .
$$

Then, if we set

$$
\begin{gathered}
\left(\bar{A}_{i+1} \backslash A_{i}\right)^{\varepsilon, \xi}:=\left\{x=y+t \xi,|t| \leq \varepsilon, y \in \bar{A}_{i+1} \backslash A_{i}\right\}, \\
S_{i}^{\varepsilon, \xi}:=\left(\bar{A}_{i+1} \backslash A_{i}\right)^{\varepsilon, \xi} \cap A,
\end{gathered}
$$

we get

$$
R_{\varepsilon}^{\xi}(A) \subseteq R_{\varepsilon}^{\xi}\left(A_{i}\right) \cup R_{\varepsilon}^{\xi}\left(A \backslash \bar{A}_{i+1}\right) \cup R_{\varepsilon}^{\xi}\left(S_{i}^{\varepsilon, \xi}\right) .
$$

Let $M_{\delta}>0$ be such that $\limsup _{\varepsilon^{+} \rightarrow 0} \sum_{|\xi|>M_{\delta}} C_{\varepsilon}^{\xi}<\delta$. Summing on $\xi \in \mathbb{Z}^{N}$, using 
(H2), (H3) and the previous decomposition, there holds

$$
\begin{aligned}
F_{\varepsilon}\left(w_{\varepsilon}^{i}, A\right) \leq & F_{\varepsilon}\left(u_{\varepsilon}, A^{\prime}\right)+F_{\varepsilon}\left(v_{\varepsilon}, A \backslash \overline{A^{\prime \prime}}\right) \\
& +C \sum_{|\xi| \leq M_{\delta}} C_{\varepsilon}^{\xi} \sum_{\alpha \in R_{\varepsilon}^{\xi}\left(S_{i}^{\varepsilon, \xi}\right)} \varepsilon^{N}\left(\left|u_{\varepsilon}(\alpha)\right|^{p}+\left|u_{\varepsilon}(\alpha+\varepsilon \xi)\right|^{p}+\left|v_{\varepsilon}(\alpha)\right|^{p}+\left|v_{\varepsilon}(\alpha+\varepsilon \xi)\right|^{p}\right) \\
& +C \sum_{|\xi|>M_{\delta}} C_{\varepsilon}^{\xi} \sum_{\alpha \in A}^{N} \varepsilon^{N}\left(\left|u_{\varepsilon}(\alpha)\right|^{p}+\left|u_{\varepsilon}(\alpha+\varepsilon \xi)\right|^{p}+\left|v_{\varepsilon}(\alpha)\right|^{p}+\left|v_{\varepsilon}(\alpha+\varepsilon \xi)\right|^{p}\right) \\
\leq & F_{\varepsilon}\left(u_{\varepsilon}, A^{\prime}\right)+F_{\varepsilon}\left(v_{\varepsilon}, A \backslash \overline{A^{\prime \prime}}\right) \\
& +C \sum_{|\xi| \leq M_{\delta}} C_{\varepsilon}^{\xi} \sum_{\alpha \in R_{\varepsilon}^{\xi}\left(S_{i}^{\varepsilon, \xi}\right)} \varepsilon^{N}\left(\left|u_{\varepsilon}(\alpha)\right|^{p}+\left|v_{\varepsilon}(\alpha)\right|^{p}\right) \\
& +C\left(\sum_{|\xi|>M_{\delta}} C_{\varepsilon}^{\xi}\right)\left(\left\|u_{\varepsilon}\right\|_{L^{p}(A)}^{p}+\left\|v_{\varepsilon}\right\|_{L^{p}(A)}^{p}\right)
\end{aligned}
$$

by symmetry of the interactions. Note that, for $\varepsilon$ small enough and $|\xi| \leq M_{\delta}$,

$R_{\varepsilon}^{\xi}\left(S_{i}^{\varepsilon, \xi}\right) \cap R_{\varepsilon}^{\xi}\left(S_{j}^{\varepsilon, \xi}\right) \neq \emptyset$ if and only if $|i-j|=1$. Note also that $\cup_{i=1}^{M-3} R_{\varepsilon}^{\xi}\left(S_{i}^{\varepsilon, \xi}\right) \subseteq R_{\varepsilon}^{\xi}\left(A^{\prime} \backslash \overline{A^{\prime \prime}}\right)$.

Thus, summing over $i \in\{1,2, \cdots, M-3\}$, averaging and taking into account (3.18) and (H3), we get

$$
\begin{aligned}
\frac{1}{M-3} \sum_{i=1}^{M-3} F_{\varepsilon}\left(w_{\varepsilon}^{i}, A\right) \leq & F_{\varepsilon}\left(u_{\varepsilon}, A^{\prime}\right)+C \delta \\
& +\frac{1}{M-3} C\left(\sum_{|\xi| \leq M_{\delta}} C_{\varepsilon}^{\xi}\right)\left(\left\|u_{\varepsilon}\right\|_{L^{p}(\Omega)}^{p}+\left\|v_{\varepsilon}\right\|_{L^{p}(\Omega)}^{p}\right) \\
& +C \delta\left(\left\|u_{\varepsilon}\right\|_{L^{p}(\Omega)}^{p}+\left\|v_{\varepsilon}\right\|_{L^{p}(\Omega)}^{p}\right)
\end{aligned}
$$

For all $M$ and $\varepsilon$ we can choose $i(\varepsilon) \in\{1,2, \cdots, M-3\}$ such that

$$
F_{\varepsilon}\left(w_{\varepsilon}^{i(\varepsilon)}, A\right) \leq \frac{1}{M-3} \sum_{j=1}^{M-3} F_{\varepsilon}\left(w_{\varepsilon}^{j}, A\right) .
$$

As $w_{\varepsilon}^{i(\varepsilon)}$ still weakly converges to $u$ in $L^{p}(\Omega)$, letting $\varepsilon$ go to zero, we obtain

$$
F^{\prime \prime}(u, A) \leq \sup _{A^{\prime} \subset \subset A} F^{\prime \prime}\left(u, A^{\prime}\right)+C\left(\frac{1}{M-3}+\delta\right) .
$$

Letting $\delta$ go to zero and $M$ to infinity concludes the proof of the thesis.

Remark 3.10 Using the same arguments as in the proof of Proposition 3.9 one can show that

$$
\begin{aligned}
& F^{\prime}(u, A)=\Gamma-\liminf _{\varepsilon \rightarrow 0^{+}} F_{\varepsilon}(u, A)=\inf \left\{\liminf _{\varepsilon \rightarrow 0^{+}} F_{\varepsilon}\left(u_{\varepsilon}, A\right): u_{\varepsilon} \rightarrow u w-L^{p}(A)\right\}, \\
& F^{\prime \prime}(u, A)=\Gamma-\limsup _{\varepsilon \rightarrow 0^{+}} F_{\varepsilon}(u, A)=\inf \left\{\limsup _{\varepsilon \rightarrow 0^{+}} F_{\varepsilon}\left(u_{\varepsilon}, A\right): u_{\varepsilon} \rightarrow u w-L^{p}(A)\right\} .
\end{aligned}
$$


Proposition 3.11 Let $\left\{f_{\varepsilon}^{\xi}\right\}$ satisfy (H1)-(H3) and be such that $f_{\varepsilon}^{\xi} \geq 0$. If $u \in$ $L^{p}(\Omega)$ and $A, B \in \mathcal{A}^{\mathcal{R}}(\Omega)$ then there holds

$$
F^{\prime \prime}(u, A \cup B) \leq F^{\prime \prime}(u, A)+F^{\prime \prime}(u, B) .
$$

If $A \cap B=\emptyset$ then

$$
F^{\prime \prime}(u, A \cup B) \geq F^{\prime \prime}(u, A)+F^{\prime \prime}(u, B) .
$$

Proof. Using the same strategy as for the proof of Proposition 3.9, we may prove that for all $A^{\prime}, B^{\prime} \in \mathcal{A}(\Omega)$ such that $A^{\prime} \subset \subset A$ and $B^{\prime} \subset \subset B$ we have

$$
F^{\prime \prime}\left(u, A^{\prime} \cup B^{\prime}\right) \leq F^{\prime \prime}(u, A)+F^{\prime \prime}(u, B) .
$$

Since for all $C \in \mathcal{A}(\Omega)$ such that $C \subset \subset A \cup B$ there exist $A^{\prime}, B^{\prime} \in \mathcal{A}(\Omega)$ such that $A^{\prime} \subset \subset A, B^{\prime} \subset \subset B$ and $C \subset A^{\prime} \cup B^{\prime}$, Proposition 3.9 shows that (3.22) implies (3.20). In addition, $F_{\varepsilon}(u, \cdot)$ is clearly superadditive, and so is $F^{\prime \prime}$ at the limit.

Proposition 3.12 Let $\left\{f_{\varepsilon}^{\xi}\right\}$ satisfy (H1)-(H3) and be such that $f_{\varepsilon}^{\xi} \geq 0$. Let $F$ be $a \Gamma$-limit of $F_{\varepsilon}$ for the weak convergence of $L^{p}(\Omega)$. Then for all $A \in \mathcal{A}^{\mathcal{R}}(\Omega)$ and $u, v \in L^{p}(\Omega)$ such that $v=u$ almost everywhere on $A$, one has

$$
F(u, A)=F(v, A) .
$$

Proof. Let $u$ and $v \in L^{p}(\Omega)$ be such that $u_{\mid A}=v_{\mid A}$ almost everywhere on $A \in \mathcal{A}(\Omega)$. As $F(\cdot, A)$ is a $\Gamma$-limit of $F_{\varepsilon}(\cdot, A)$, we have that for all $w_{\varepsilon} \rightarrow v$ and $\tilde{w}_{\varepsilon} \rightarrow u$ in $L^{p}(A), F(u, A) \leq \liminf F_{\varepsilon}\left(w_{\varepsilon}, A\right)$ and $F(v, A) \leq \liminf F_{\varepsilon}\left(w_{\varepsilon}, A\right)$.

Let now $u_{\varepsilon}$ and $v_{\varepsilon}$ be recovery sequences for $F(u, A)$ and $F(v, A)$ in $L^{p}(A)$. As $u_{\mid A}=v_{\mid A}$ almost everywhere on $A$, one also has $v_{\varepsilon} \rightarrow u_{\mid A}$ in $L^{p}(A)$ and $u_{\varepsilon} \rightarrow v_{\mid A}$ in $L^{p}(A)$. Thus, $F(v, A) \leq \liminf F_{\varepsilon}\left(u_{\varepsilon}, A\right)=F(u, A)$ and $F(u, A) \leq$ $\liminf F_{\varepsilon}\left(v_{\varepsilon}, A\right)=F(v, A)$, which shows the thesis.

Proof of Theorem 3.1 By Remark 3.8 it is not restrictive to suppose $f_{\varepsilon}^{\xi} \geq 0$. To conclude we first need to use the compactness of $\Gamma$-convergence w.r.t. weak topologies. To this end we observe that, if we define

$$
\tilde{F}_{\varepsilon}(u, A)= \begin{cases}F_{\varepsilon}(u, A) & \text { if } u \in C_{\varepsilon}\left(A, \mathbb{R}^{m}\right), \\ & u(\alpha)=0 \text { if } \alpha+[0, \varepsilon]^{N} \cap \partial A \neq \emptyset \\ +\infty & \text { otherwise, }\end{cases}
$$

then, by using the same argument exploited in the proof of Theorem 3.9, it can be shown that $\tilde{F}^{\prime}(u, A)=F^{\prime}(u, A)$ and that $\tilde{F}^{\prime \prime}(u, A)=F^{\prime \prime}(u, A)$. Moreover $\tilde{F}_{\varepsilon}(u, A) \geq c\left(\|u\|_{L^{p}(A)}^{p}-1\right)$ for some constant $c>0$. Then by Corollary 8.12 in [16], Proposition 3.9 and Theorem 10.3 in [10], there exists a subsequence $\varepsilon_{j_{k}}$ such that, for all $(u, A) \in L^{p}(\Omega) \times \mathcal{A}^{\mathcal{R}}(\Omega)$, there exists

$$
\Gamma\left(w-L^{p}\right)-\lim _{k} F_{\varepsilon_{j_{k}}}(u, A)=: F(u, A) .
$$


Moreover we can extend $F(u, \cdot)$ to $\mathcal{A}(\Omega)$ by setting $F(u, A)=\sup \left\{F\left(u, A^{\prime}\right): A^{\prime} \in\right.$ $\left.\mathcal{A}^{\mathcal{R}}(\Omega), A^{\prime} \subset \subset A\right\}$ and easily verify that all the results contained in Propositions $3.6,3.7,3.9,3.11$ and 3.12 still hold true. Hence, by the De Giorgi-Letta Criterion (see [10]), $F(u, \cdot)$ is the restriction on $\mathcal{A}(\Omega)$ of a Borel measure $\bar{F}(u, \cdot)$ which, by Proposition 3.7, is absolutely continuous w.r.t. $\mathcal{L}^{N}$. By the lower semicontinuity of $F(u, A)$ and standard arguments in measure theory, $\bar{F}(u, \cdot)$ fulfills all the hypotheses of Theorem 2.1, by which we get the conclusion.

The proof of the previous theorem actually shows that a local version of Theorem 2.1 holds

Theorem 3.13 Let $F_{\varepsilon}$ be as in (3.6), and $\left\{f_{\varepsilon}^{\xi}\right\}_{\varepsilon, \xi}$ satisfy hypotheses (H1), (H2) and (H3). Let $\left(\varepsilon_{j}\right)$ and $f$ be as in Theorem 3.1. Then, for any $u \in L^{p}\left(\Omega, \mathbb{R}^{m}\right)$ and $A \in \mathcal{A}(\Omega)$, there holds

$$
\Gamma\left(w-L^{p}\right)-\lim _{j} F_{\varepsilon_{j}}(u, A)=\int_{A} f(x, u(x)) d x .
$$

\subsection{Proof in $L^{\infty}$}

The proof of Theorem 3.3 is an easy adaptation of the proof of Theorem 3.1. Let $F^{\prime}(u, A)$ and $F^{\prime \prime}(u, A)$ be given by $(3.13)$ (note that on $L^{\infty}(\Omega, \bar{K})$ the weak $L^{p}$ topologies are all equivalent for any $p)$. Moreover note that for any $u_{\varepsilon} \in C_{\varepsilon}(\Omega, K)$ such that $u_{\varepsilon} \rightarrow^{*} u$ in $L^{\infty}$ then $u \in L^{\infty}(\Omega, \bar{K})$, and, reciprocally, for any $u \in$ $L^{\infty}(\Omega, \bar{K})$ one can construct $u_{\varepsilon} \in C_{\varepsilon}(\Omega, K)$ such that $u_{\varepsilon}-^{*} u$ in $L^{\infty}$. By $(H 5)$ it holds that, for any $u \in C_{\varepsilon}(\Omega, K)$ and $A \in \mathcal{A}^{R}(\Omega)$

$$
\begin{aligned}
\left|F_{\varepsilon}(u, A)\right| & \leq \sum_{\xi \in \mathbb{Z}^{N}} \sum_{\alpha \in R_{\varepsilon}^{\xi}(A)} \varepsilon^{N} C_{\varepsilon, \alpha}^{\xi} \\
& =\sum_{\alpha \in A} \sum_{\xi \in \mathbb{Z}^{N}} \varepsilon^{N} C_{\varepsilon, \alpha}^{\xi} \\
& \leq C(|A|+O(\varepsilon)) .
\end{aligned}
$$

Then, by (H4) and (3.23) we get that $F^{\prime}(u, A)$ and $F^{\prime \prime}(u, A)$ are finite if and only if $u \in L^{\infty}(\Omega, \bar{K})$ and satisfy

$$
-C|A| \leq F^{\prime}(u, A) \leq F^{\prime \prime}(u, A) \leq C|A| .
$$

All the properties stated in Propositions 3.9, 3.11 and 3.12 hold true in the present case for any $u, v \in L^{\infty}(\Omega, \bar{K})$ and for all $A \in \mathcal{A}^{R}(\Omega)$, the proof being the same.

Since the weak topology on $L^{\infty}(\Omega, \bar{K})$ is metrizable, by the compactness property of $\Gamma$-convergence in metric spaces, there exists a subsequence $\varepsilon_{j} \rightarrow 0$ such that, for any $(u, A) \in L^{\infty}(\Omega, \bar{K}) \times \mathcal{A}^{R}(\Omega)$

$$
\Gamma\left(w^{*}-L^{\infty}\right)-\lim _{j} F_{\varepsilon_{j}}(u, A)=F(u, A) .
$$


As in the proof of the $L^{p}$ case, we may extend $F(u, \cdot)$ to $\mathcal{B}(\Omega)$. Then, by applying Theorem 2.1 to the functional $\bar{F}: L^{p}\left(\Omega, \mathbb{R}^{m}\right) \times \mathcal{B}(\Omega) \rightarrow[0,+\infty]$ defined by

$$
\bar{F}(u, B)= \begin{cases}F(u, B) & \text { if } u \in L^{\infty}(\Omega, \bar{K}) \\ +\infty & \text { otherwise }\end{cases}
$$

we get the conclusion.

As in the $L^{p}$ case the following local version of Theorem 3.3 holds true:

Theorem 3.14 Let $F_{\varepsilon}$ be as in (3.6), and $\left\{f_{\varepsilon}^{\xi}\right\}_{\varepsilon, \xi}$ satisfy hypotheses (H4), (H5) and (H6). Let $\left(\varepsilon_{j}\right)$ and $f$ be as in Theorem 3.3. Then, for any $u \in L^{\infty}(\Omega, \bar{K})$ and $A \in \mathcal{A}(\Omega)$, there holds

$$
\Gamma\left(w^{*}-L^{\infty}\right)-\lim _{j} F_{\varepsilon_{j}}(u, A)=\int_{A} f(x, u(x)) d x .
$$

\section{Minimum problems}

In this section we derive a convergence result for minimum problems in the case that our functionals are subject to mean type constraints. Let us introduce the notion of discrete mean.

Definition 4.1 For any $A \subset \Omega, \varepsilon>0$, and $u \in C_{\varepsilon}\left(\Omega, \mathbb{R}^{m}\right)$, we set

$$
\langle u\rangle_{A}^{d, \varepsilon}=\frac{1}{\#\left(\varepsilon \mathbb{Z}^{N} \cap A\right)} \sum_{\alpha \in \varepsilon \mathbb{Z}^{N} \cap A} u(\alpha) .
$$

Given $z \in \mathbb{R}^{m}$, we define $F_{\varepsilon}^{z}: L^{p}(\Omega) \times \mathcal{A}(\Omega) \rightarrow(-\infty,+\infty]$ by

$$
F_{\varepsilon}^{z}(u, A)= \begin{cases}F_{\varepsilon}(u, A) & \langle u\rangle_{A}^{\varepsilon, d}=z \\ +\infty & \text { otherwise. }\end{cases}
$$

The following theorem holds true.

Theorem 4.2 Let $\left\{f_{\varepsilon}^{\xi}\right\}$ satisfy hypotheses (H1), (H2) and (H3). Let $\left(\varepsilon_{j}\right)$ and $f$ be as in Theorem 3.1. For any $z \in \mathbb{R}^{m}$, let $F_{\varepsilon_{j}}^{z}$ be as in (4.26). Then, for any $A \in \mathcal{A}^{R}(\Omega),\left(F_{\varepsilon_{j}}^{z}(\cdot, A)\right) \Gamma$-converges with respect to the weak convergence of $L^{p}\left(\Omega, \mathbb{R}^{m}\right)$ to the functional $F^{z}: L^{p}(\Omega) \times \mathcal{A}^{R}(\Omega) \rightarrow(-\infty,+\infty]$ defined by

$$
F^{z}(u, A)= \begin{cases}\int_{A} f(x, u) d x & \langle u\rangle_{A}=z \\ +\infty & \text { otherwise }\end{cases}
$$


Proof. Let us first prove the lower bound inequality. Let $A \in \mathcal{A}^{R}(\Omega)$ and let $\left(u_{j}\right)$ be a sequence of functions converging to $u$ w.r.t. the weak convergence of $L^{p}(\Omega)$ such that

$$
\liminf _{j} F_{\varepsilon_{j}}^{z}\left(u_{j}, A\right)=\lim _{j} F_{\varepsilon_{j}}^{z}\left(u_{j}, A\right)<+\infty .
$$

As $\left\langle u_{j}\right\rangle_{A}^{\varepsilon_{j}, d}=z$ and, by the equi-integrability of $u_{j}$ we get $\langle u\rangle_{A}=z$. Th lower bound inequality follows by Theorem 3.13, observing that

$$
F_{\varepsilon_{j}}^{z}\left(u_{j}, A\right) \geq F_{\varepsilon_{j}}\left(u_{j}, A\right) .
$$

To prove the upper bound inequality let us observe that, fixed $z \in \mathbb{R}^{m}$ and $u \in L^{p}(\Omega)$ such that $\langle u\rangle_{A}=z$, by using the argument exploited in the proof of Proposition 3.9, for every $\delta>0$ there exists $B \subset \subset A$ and a sequence of functions $u_{j} \rightarrow u$ weakly in $L^{p}(\Omega)$ such that

$$
\begin{aligned}
& \limsup _{j} F_{\varepsilon_{j}}\left(u_{j}, A\right) \leq F(u, A)+\delta, \\
& \limsup _{j} \sum_{\alpha \in R_{\varepsilon}^{\xi}(A \backslash \bar{B})} \varepsilon^{N}\left(\left|u_{j}(\alpha)\right|^{p}+D_{\varepsilon}(\alpha)\right) \leq C \delta
\end{aligned}
$$

for some constant $C>0$. Set $z_{j}=\left\langle u_{j}\right\rangle_{A}^{\varepsilon_{j}, d}$ and let $B^{\prime}$ be such that $B \subset \subset B^{\prime} \subset \subset A$. We then define

$$
v_{j}(\alpha)= \begin{cases}u_{j}(\alpha) & \alpha \in \varepsilon_{j} \mathbb{Z}^{N} \cap B^{\prime} \\ u_{j}(\alpha)+c_{j} & \alpha \in \varepsilon_{j} \mathbb{Z}^{N} \cap\left(A \backslash \overline{B^{\prime}}\right),\end{cases}
$$

where

$$
c_{j}=\left(z-z_{j}\right) \frac{\#\left(\varepsilon_{j} \mathbb{Z}^{N} \cap A\right)}{\#\left(\varepsilon_{j} \mathbb{Z}^{N} \cap\left(A \backslash \overline{B^{\prime}}\right)\right.} .
$$

Then, $\left\langle v_{j}\right\rangle_{A}^{\varepsilon_{j}, d}=z$ and, since $z_{j} \rightarrow z$, we have that $v_{j} \rightarrow u$ weakly in $L^{p}(A)$. By (4.27), since $c_{j} \rightarrow 0$, we conclude that

$$
\limsup _{j} F_{\varepsilon_{j}}^{z}\left(v_{j}, A\right) \leq F^{z}(u, A)+\delta .
$$

By letting $\delta$ go to 0 we obtain the claim.

Remark 4.3 For all $\eta>0$ set $A_{\eta}=\{x \in A \mid d(x, \partial A)>\eta\}$. The proof of the previous result shows that, if, for every $L>0$, we replace the functional $F_{\varepsilon}^{z}(u, A)$ in (4.26) by

$$
F_{\varepsilon}^{z}(u, A)= \begin{cases}F_{\varepsilon}(u, A) & \langle u\rangle_{A}^{\varepsilon, d}=z \text { and } u(\alpha)=z \text { if } \alpha \in A \backslash A_{\varepsilon L} \\ +\infty & \text { otherwise, }\end{cases}
$$

then the conclusion of Theorem 4.2 still holds true. 
By the equicoercivity of the energies $F_{\varepsilon}^{z}$ and the properties of $\Gamma$-convergence we derive the following corollary

Corollary 4.4 Under the hypotheses of Theorem 4.2, for any $z \in \mathbb{R}^{m}, A \in \mathcal{A}^{R}(\Omega)$ and for L large enough,

$$
\begin{aligned}
& \liminf _{j}\left\{F_{\varepsilon_{j}}(u, A):\langle u\rangle_{A}^{\varepsilon, d}=z \text { and } u(\alpha)=z \text { if } \alpha \in A \backslash A_{\varepsilon L}\right\} \\
& =\min \left\{F(u, A):\langle u\rangle_{A}=z\right\} .
\end{aligned}
$$

In addition, if $\left(u_{j}\right)$ is a converging sequence such that

$\lim _{j} F_{\varepsilon_{j}}\left(u_{j}, A\right)=\lim _{j} \inf \left\{F_{\varepsilon_{j}}(u, A):\langle u\rangle_{A}^{\varepsilon, d}=z\right.$ and $u(\alpha)=z$ if $\left.\alpha \in A \backslash A_{\varepsilon L}\right\}$,

then its limit is a solution of $\min \left\{F(u, A):\langle u\rangle_{A}=z\right\}$.

Proof. It suffices to observe that, by the coercivity assumption (H1), for $L$ large enough, the minimizing sequence $u_{j}$ is bounded in the $L^{p}$-norm. The conclusion follows by Theorem 4.2 and the properties of $\Gamma$-convergence.

In the $L^{\infty}$ case, due to the discrete structure of the problem and the fact that the functions in the domain of $F_{\varepsilon}$ take values in a set which will be relaxed in the limit procedure, one need to relax the constraint at the discrete level and consider, for all $z \in \mathbb{R}^{m}$ and $\rho>0$, the functional $F_{\varepsilon}^{z, \rho}: L^{\infty}(\Omega) \times \mathcal{A}(\Omega) \rightarrow \mathbb{R}$ given by

$$
F_{\varepsilon}^{z, \rho}(u, A)= \begin{cases}F_{\varepsilon}(u, A) & \langle u\rangle_{A}^{\varepsilon, d} \in \bar{B}(z, \rho) \\ +\infty & \text { otherwise }\end{cases}
$$

with $F_{\varepsilon}$ as in (3.6). The following $\Gamma$-convergence result holds true.

Theorem 4.5 Let $\left\{f_{\varepsilon}^{\xi}\right\}$ satisfy hypotheses (H4), (H5) and (H6). Let $\left(\varepsilon_{j}\right)$ and $f$ be as in Theorem 3.3. Then, for any $z \in \bar{K}, \rho>0$ and $A \in \mathcal{A}^{R}(\Omega)\left(F_{\varepsilon_{j}}^{z, \rho}(\cdot, A)\right)$ $\Gamma$-converges with respect to the weak ${ }^{*}$-convergence of $L^{\infty}\left(\Omega, \mathbb{R}^{m}\right)$ to the functional $F^{z, \rho}: L^{\infty}(\Omega, \bar{K}) \times \mathcal{A}^{R}(\Omega) \rightarrow(-\infty,+\infty]$ given by

$$
F^{z, \rho}(u, A)= \begin{cases}\int_{A} f(x, u) d x & u \in L^{\infty}(A ; \bar{K}),\langle u\rangle_{A} \in \bar{B}(z, \rho) \\ +\infty & \text { otherwise. }\end{cases}
$$

Proof. The lower bound inequality is straightforward thanks to Theorem 3.3, observing that the constraint is closed under weak *-convergence. By density it is enough to prove the upper bound inequality for $u$ such that $\langle u\rangle_{A} \in B(z, \rho)$. For such a $u$ we conclude by observing that the optimizing sequence $u_{j}$ for $\int_{A} f(x, u(x)) d x$ satisfies the constraint $\langle u\rangle_{A}^{\varepsilon_{j}, d} \in B(z, \rho)$ for $j$ large enough.

By the properties of $\Gamma$-convergence, the previous theorem yields the following result about the convergence of minimum problems. 
Corollary 4.6 Under the hypotheses of Theorem 4.5, for any $z \in \mathbb{R}^{m}, \rho>0$ and $A \in \mathcal{A}^{R}(\Omega)$,

$$
\lim _{j} \inf \left\{F_{\varepsilon_{j}}(u, A):\langle u\rangle_{A}^{\varepsilon_{j}, d} \in \bar{B}(z, \rho)\right\}=\min \left\{F(u, A):\langle u\rangle_{A} \in \bar{B}(z, \rho)\right\} .
$$

In addition, if $\left(u_{j}\right)$ is a converging sequence such that

$$
\lim _{j} F_{\varepsilon_{j}}\left(u_{j}, A\right)=\lim _{\rho} \liminf _{j}\left\{F_{\varepsilon_{j}}(u, A):\langle u\rangle_{A}^{\varepsilon_{j}, d} \in \bar{B}(z, \rho)\right\},
$$

then its limit is a solution of $\min \left\{F(u, A):\langle u\rangle_{A} \in \bar{B}(z, \rho)\right\}$.

\section{Homogenization}

In this section we show that if the energy densities $f_{\varepsilon}^{\xi}$ are obtained by scaling periodic functions $f^{\xi}$ by $\varepsilon$ in the space variable, then the energy density of the limit functional does not depend on the space variable and is given by a homogenization formula.

\subsection{Homogenization in $L^{p}, 1<p<\infty$}

Let $k \in \mathbb{N}$ and for any $\xi \in \mathbb{Z}^{N}$, let $f^{\xi}: \mathbb{Z}^{N} \times \mathbb{R}^{m} \times \mathbb{R}^{m} \rightarrow \mathbb{R}$ be such that $f^{\xi}(\cdot, u, v)$ is $[0, k]^{N}$-periodic for any $u, v \in \mathbb{R}^{m}$. We then set

$$
f_{\varepsilon}^{\xi}(\alpha, u, v):=f^{\xi}\left(\frac{\alpha}{\varepsilon}, u, v\right) .
$$

In this case, hypotheses (H1), (H2), (H3) read

(H7) For all $\alpha$ and $\xi$ there exist $c^{\xi} \geq 0$ and $d^{\xi} \geq 0$ such that

$$
f^{\xi}(\alpha, u, v) \geq c^{\xi}\left(|u|^{p}+|v|^{p}\right)-d^{\xi}
$$

for all $(u, v) \in \mathbb{R}^{2 m}$, there exists $\bar{\xi} \in \mathbb{Z}^{N}$ with $c^{\bar{\xi}}>0$, and $\sum_{\xi} d^{\xi}<\infty$.

(H8) For all $\alpha$ and $\xi$, there exists $C^{\xi} \geq 0$ such that

$$
f^{\xi}(\alpha, u, v) \leq C^{\xi}\left(|u|^{p}+|v|^{p}+1\right)
$$

for all $(u, v) \in \mathbb{R}^{2 m}$, and $\sum_{\xi} C^{\xi}<\infty$.

In what follows, for simplicity of notation, we will write $\langle u\rangle_{A}^{d}$ instead of $\langle u\rangle_{A}^{d, 1}$. We have the following

Theorem 5.1 Let $\left\{f_{\varepsilon}^{\xi}\right\}_{\varepsilon, \xi}$ satisfy (5.31), (H $\left.H^{\eta}\right)$ and (H8). Then $F_{\varepsilon} \Gamma\left(w-L^{p}\right)$ converges to

$$
F(u)=\int_{\Omega} f_{h o m}(u(x)) d x
$$


for all $u \in L^{p}(\Omega)$, where $f_{\text {hom }}$ is given by the homogenization formula

$$
f_{\text {hom }}(z)=\lim _{h \rightarrow+\infty} \frac{1}{h^{N}} \inf \left\{\sum_{\xi \in \mathbb{Z}^{N}} \sum_{\beta \in R_{1}^{\xi}\left(Q_{h}\right)} f^{\xi}(\beta, v(\beta), v(\beta+\xi)),\langle v\rangle_{Q_{h}}^{d}=z\right\}
$$

and $Q_{h}=(0, h)^{N}$.

Proof. Let $\left(\varepsilon_{n}\right)$ be a sequence of positive numbers converging to 0 . Then, by Theorem 3.13 , we can extract a subsequence (not relabeled) such that $\left(F_{\varepsilon_{n}}(\cdot, A)\right)$ $\Gamma$-converges to a functional $F(\cdot, A)$ defined as in (3.8). The theorem is proved if we show that the density function $f$ does not depend on the space variable $x$ and if $f \equiv f_{\text {hom }}$.

To prove the independence upon the space variable, it suffices to show that

$$
F(z, B(x, \rho))=F(z, B(y, \rho))
$$

for all $x, y \in \Omega, \rho>0$ and $z \in \mathbb{R}^{m}$. Using the inner regularity and by changing the roles of $x$ and $y$, it suffices to have

$$
F\left(z, B\left(x, \rho^{\prime}\right)\right) \leq F(z, B(y, \rho))
$$

for all $\rho^{\prime}<\rho$. Let $v_{n} \rightarrow z$ in $L^{p}(\Omega)$ be such that

$$
\lim _{n} F_{\varepsilon_{n}}\left(v_{n}, B(y, \rho)\right)=F(z, B(y, \rho)) .
$$

Then set

$$
u_{n}(\alpha)= \begin{cases}v_{n}\left(\alpha-\varepsilon_{n}\left[\frac{x-y}{\varepsilon_{n}}\right]_{k}\right) & \text { if } \alpha \in \varepsilon_{n} \mathbb{Z}^{N} \cap B\left(x, \rho^{\prime}\right) \\ z & \text { otherwise }\end{cases}
$$

Due to the periodicity (5.31), for $n$ large enough, we have

$$
F_{\varepsilon_{n}}\left(u_{n}, B\left(x, \rho^{\prime}\right)\right) \leq F_{\varepsilon_{n}}\left(v_{n}, B(y, \rho)\right) .
$$

From this, we easily get (5.33) since $u_{n} \rightarrow z$.

The second step consists in proving that $f \equiv f_{\text {hom }}$. To this end, we note that, since $f(\cdot)$ is a convex function, there holds

$$
\begin{aligned}
f(z) & =\frac{1}{r^{N}} \min \left\{\int_{Q_{r}} f(u) d x,\langle u\rangle_{Q_{r}}=z\right\} \\
& =\lim _{n} \frac{1}{r^{N}} \inf \left\{F_{\varepsilon_{n}}\left(u, Q_{r}\right),\langle u\rangle_{Q_{r}}^{d, \varepsilon_{n}}=z\right\} .
\end{aligned}
$$


The second equality is a consequence of the convergence of minima given by Corollary 4.4. Set $h_{n}=\left[\frac{r}{\varepsilon_{n}}\right]+1$, then (5.34) holds with $\varepsilon_{n} h_{n}$ instead of $r$. Eventually, through the change of variable

$$
\beta=\frac{\alpha}{\varepsilon}, \quad v(\beta)=u(\varepsilon \beta),
$$

we get

$$
f(z)=\lim _{n} \frac{1}{h_{n}^{N}} \inf \left\{\sum_{\xi \in \mathbb{Z}^{N}} \sum_{\beta \in R_{1}^{\xi}\left(Q_{h_{n}}\right)} f^{\xi}(\beta, v(\beta), v(\beta+\xi)),\langle v\rangle_{Q_{h_{n}}}^{d}=z\right\} .
$$

One then infers the thesis from the existence of $\lim _{n \rightarrow \infty} I(n, z)$, where

$$
I(n, z)=\frac{1}{n^{N}} \inf \left\{\sum_{\xi \in \mathbb{Z}^{N}} \sum_{\beta \in R_{1}^{\xi}\left(Q_{n}\right)} f^{\xi}(\beta, v(\beta), v(\beta+\xi)),\langle v\rangle_{Q_{n}}^{d}=z\right\} .
$$

To prove the existence of this limit, let us first truncate the range of the interactions and define for any $R>0$,

$$
F_{1}^{R}\left(u, Q_{n}\right)=\sum_{\xi \in \mathbb{Z}^{N},|\xi|<R} \sum_{\beta \in R_{1}^{\xi}\left(Q_{n}\right)} f^{\xi}(\beta, v(\beta), v(\beta+\xi)),
$$

and

$$
I^{R}(n, z)=\frac{1}{n^{N}} \inf \left\{F_{1}^{R}\left(u, Q_{n}\right),\langle v\rangle_{Q_{n}}=z\right\} .
$$

Using (H8) one can prove that

$$
\lim _{R \rightarrow \infty} \sup _{n}\left|I^{R}(n, z)-I(n, z)\right|=0 .
$$

For $n>R$, let us introduce

$$
I^{R, R}(n, z)=\frac{1}{n^{N}} \inf \left\{F_{1}^{R}\left(u, Q_{n}\right),\langle v\rangle_{Q_{n}}=z, v(\beta)=z \forall \beta \in Q_{n} \backslash Q_{n-R}\right\} .
$$

By using the same arguments as for Theorem 4.2, thanks to Remark 4.3 and Corollary 4.4 , for any sequence $n_{h} \rightarrow+\infty$ there exists a subsequence (not relabelled) such that

$$
\lim _{h} I^{R}\left(n_{h}, z\right)=\lim _{h} I^{R, R}\left(n_{h}, z\right) .
$$

It is then enough to prove that $\lim _{n \rightarrow \infty} I^{R, R}(n, z)$ exists for all $z \in \mathbb{R}^{m}$. 
To this end let $n \in \mathbb{N}$ and let $v_{n}$ be a test function for the minimum problem defining $I^{R, R}(n, z)$ such that

$$
\frac{1}{n^{N}} F_{1}^{R}\left(v_{n}, Q_{n}\right) \leq I^{R, R}(n, z)+\frac{1}{n} .
$$

We then define, for any $k>n$, a test function $u_{k}$ in the minimum problem defining $I^{R, R}(k, z)$ as follows:

$$
u_{k}(\beta)= \begin{cases}v_{n}(\beta-n i) & \text { if } \beta \in n i+Q_{n}, \quad i \in\left\{0, \ldots,\left[\frac{k}{n}\right]-1\right\}^{N} \\ z & \text { otherwise. }\end{cases}
$$

By the growth hypotheses on $f^{\xi}$ and the constancy of $u_{k}$ near the boundary of $Q_{n}$, we get

$$
\begin{aligned}
I_{k}^{R, R}(z) \leq & \frac{1}{k^{N}} F_{1}^{R}\left(u_{k}, Q_{k}\right) \leq\left[\frac{k}{n}\right]^{N} \frac{1}{k^{N}} F_{1}^{R}\left(v_{n}, Q_{n}\right) \\
& +C|z|^{p} \frac{1}{k^{N}}\left(k^{N}-\left[\frac{k}{n}\right]^{N} n^{N}+\left[\frac{k}{n}\right]^{N}\left((n+R)^{N}-(n-R)^{N}\right)\right) \\
& \leq\left[\frac{k}{n}\right]^{N} \frac{n^{N}}{k^{N}}\left(I_{n}^{R, R}(z)+\frac{1}{n}\right) \\
& +C|z|^{p} \frac{1}{k^{N}}\left(k^{N}-\left[\frac{k}{n}\right]^{N} n^{N}+\left[\frac{k}{n}\right]^{N}\left((n+R)^{N}-(n-R)^{N}\right)\right)
\end{aligned}
$$

By letting $k$ tend to $+\infty$, we then get

$$
\limsup _{k} I_{k}^{R, R}(z) \leq I_{n}^{R, R}(z)+\frac{1}{n}+C|z|^{p} \frac{1}{n^{N}}\left((n+R)^{N}-(n-R)^{N}\right)
$$

Eventually, letting $n$ tend to $+\infty$, we obtain

$$
\limsup _{k} I_{k}^{R, R}(z) \leq \liminf _{n} I_{n}^{R, R}(z)
$$

that is the claim.

\subsubsection{The convex case}

In this subsection we prove that in the convex case the function $f_{\text {hom }}$ can be obtained by a minimization problem on one single periodic cell $Q_{k}=(0, k)^{N}$.

Theorem 5.2 Let $\left(f_{\varepsilon}^{\xi}\right)_{\varepsilon, \xi}$ satisfy the assumptions of Theorem 5.1 and in addition let $f_{\varepsilon}^{\xi}(\alpha, u, v)$ be convex w.r.t. the couple $(u, v)$ for all $\alpha \in \varepsilon \mathbb{Z}^{N}, \varepsilon>0$ and $\xi \in \mathbb{Z}^{N}$. Then the conclusion of Theorem 5.1 holds with $f_{\text {hom }}$ given by

$$
f_{\text {hom }}(z)=\frac{1}{k^{N}} \inf \left\{\sum_{\xi \in \mathbb{Z}^{N}} \sum_{\beta \in I_{k}} f^{\xi}(\beta, v(\beta), v(\beta+\xi)), \quad\langle v\rangle_{Q_{k}}^{d}=z\right\},
$$


for all $z \in \mathbb{R}^{N}$, where $I_{k}=\{0, \ldots, k-1\}^{N}$.

Proof. Set

$$
\bar{f}(z)=\frac{1}{k^{N}} \inf \left\{\sum_{\xi \in \mathbb{Z}^{N}} \sum_{\beta \in I_{k}} f^{\xi}(\beta, v(\beta), v(\beta+\xi)), \quad\langle v\rangle_{Q_{k}}^{d}=z\right\} .
$$

We first prove that

$$
f_{\text {hom }}(z) \leq \bar{f}(z) .
$$

With fixed $\delta>0$, let $v$ be such that $\langle v\rangle_{Q_{k}}^{d}=z$ and that

$$
\frac{1}{k^{N}} \sum_{\xi \in \mathbb{Z}^{N}} \sum_{\beta \in I_{k}} f^{\xi}(\beta, v(\beta), v(\beta+\xi)) \leq \bar{f}(z)+\delta .
$$

For $n \in \mathbb{N}$, let $I(n, z)$ be as in (5.36). Since in particular $\langle v\rangle_{Q_{n k}}^{d}=z$, we get

$$
\begin{aligned}
I(n k, z) & \leq \frac{1}{n^{N} k^{N}} \sum_{\xi \in \mathbb{Z}^{N}} \sum_{\beta \in R_{1}^{\xi}\left(Q_{n k}\right)} f^{\xi}(\beta, v(\beta), v(\beta+\xi)) \\
& \leq \frac{1}{k^{N}} \sum_{\xi \in \mathbb{Z}^{N}} \sum_{\beta \in I_{k}} f^{\xi}(\beta, v(\beta), v(\beta+\xi)) \leq \bar{f}(z)+\delta .
\end{aligned}
$$

Estimate (5.40) follows by letting $n$ go to $+\infty$, thanks to the arbitrariness of $\delta$. We now prove that

$$
f_{\text {hom }}(z) \geq \bar{f}(z)
$$

To this end we set

$$
\bar{f}^{R}(z)=\frac{1}{k^{N}} \inf \left\{\sum_{|\xi| \leq R} \sum_{\beta \in I_{k}} f^{\xi}(\beta, v(\beta), v(\beta+\xi)), \quad\langle v\rangle_{Q_{k}}^{d}=z\right\},
$$

and

$$
f_{\text {hom }}^{R}(z)=\lim _{n \rightarrow+\infty} I^{R, R}(n, z)
$$

where $I^{R, R}(n, z)$ is defined in (5.38). By (5.37) and (5.39) we get

$$
\lim _{R \rightarrow+\infty} f_{\text {hom }}^{R}(z)=f_{\text {hom }}(z) .
$$

Analogously one can show that

$$
\lim _{R \rightarrow+\infty} \bar{f}^{R}(z)=\bar{f}(z) .
$$

Thus it suffices to prove that, for every $R>0$

$$
f_{\text {hom }}^{R}(z) \geq \bar{f}^{R}(z) \text {. }
$$


For $n \in \mathbb{N}, n k>R$, let $v$ be such that $\langle v\rangle_{Q_{n k}}=z, v(\beta)=z \forall \beta \in Q_{n k} \backslash Q_{n k-R}$. Hence

$$
\begin{aligned}
& \frac{1}{n^{N} k^{N}} \sum_{|\xi| \leq R} \sum_{\beta \in R_{1}^{\xi}\left(Q_{n k}\right)} f^{\xi}(\beta, v(\beta), v(\beta+\xi)) \\
= & \frac{1}{n^{N} k^{N}} \sum_{|\xi| \leq R} \sum_{\beta \in I_{n k}} f^{\xi}(\beta, v(\beta), v(\beta+\xi))-O\left(\frac{1}{n}\right) \\
= & \frac{1}{k^{N}} \sum_{|\xi| \leq R} \sum_{\beta \in I_{k}} \frac{1}{n^{N}} \sum_{\gamma \in\{1, \ldots, n\}^{N}} f^{\xi}\left(\beta, v\left(\beta+k \sum_{i=1}^{N} \gamma_{i} e_{i}\right), v\left(\beta+k \sum_{i=1}^{N} \gamma_{i} e_{i}+\xi\right)\right)-O\left(\frac{1}{n}\right) \\
\geq & \frac{1}{k^{N}} \sum_{|\xi| \leq R} \sum_{\beta \in I_{k}} f^{\xi}\left(\beta, v_{n}(\beta), v_{n}(\beta+\xi)\right)-O\left(\frac{1}{n}\right),
\end{aligned}
$$

where we have set

$$
v_{n}(\beta)=\frac{1}{n_{\gamma \in\{1, \ldots, n\}^{N}}^{N}} v\left(\beta+k \sum_{i=1}^{N} \gamma_{i} e_{i}\right)
$$

and the last inequality follows by the convexity hypothesis on $f^{\xi}$. Since $\left\langle v_{n}\right\rangle_{Q_{k}}=z$, by (5.42) and the definition of $\bar{f}^{R}(z)$, we obtain

$$
\frac{1}{n^{N} k^{N}} \sum_{|\xi| \leq R} \sum_{\beta \in R_{1}^{\xi}\left(Q_{n k}\right)} f^{\xi}(\beta, v(\beta), v(\beta+\xi)) \geq \bar{f}^{R}(z)-O\left(\frac{1}{n}\right) .
$$

Taking the infimum with respect to $v$ and then letting $n$ tend to $+\infty$, we obtain (5.41).

\subsection{Homogenization in $L^{\infty}$}

Let $f_{\varepsilon}^{\xi}$ be as in (5.31) where $f^{\xi}(\cdot, u, v)$ is $[0, k]^{n}$-periodic for any $u, v \in \mathbb{R}^{m}$. In this case hypotheses $(H 4),(H 5),(H 6)$ read:

(H9) For all $\alpha$ and $\xi, f^{\xi}(\alpha, u, v)=+\infty$ if $(u, v) \notin K^{2}$.

(H10) For all $\alpha$ and $\xi$, there exists $C^{\xi} \geq 0$ such that $\left|f^{\xi}(\alpha, u, v)\right| \leq C^{\xi}$ for all $(u, v) \in K^{2}$, and $\sum_{\xi} C^{\xi}<\infty$.

The following theorem holds.

Theorem 5.3 Let $\left\{f_{\varepsilon}^{\xi}\right\}_{\varepsilon, \xi}$ satisfy (5.31), (H9) and (H10). Then $F_{\varepsilon} \Gamma\left(w *-L^{\infty}\right)$ converges to

$$
F(u)=\int_{\Omega} f_{h o m}(u(x)) d x
$$


for all $u \in L^{\infty}(\Omega ; \bar{K})$, where $f_{\text {hom }}$ is given by the homogenization formula

$f_{\text {hom }}(z)=\lim _{\rho \rightarrow 0} \lim _{h \rightarrow+\infty} \frac{1}{h^{N}} \inf \left\{\sum_{\xi \in \mathbb{Z}^{N}} \sum_{\beta \in R_{1}^{\xi}\left(Q_{h}\right)} f^{\xi}(\beta, v(\beta), v(\beta+\xi)),\langle v\rangle_{Q_{h}}^{d} \in \bar{B}(z, \rho)\right\}$.

Proof. Let $\left(\varepsilon_{n}\right)$ be a sequence of positive numbers converging to 0 . Then, by Theorem 3.14, we can extract a subsequence (not relabeled) such that $\left(F_{\varepsilon_{n}}(\cdot, A)\right)$ $\Gamma$-converges to a functional $F(\cdot, A)$ defined as in (3.9). The theorem is proved if we show that the density function $f$ does not depend on the space variable $x$ and if $f \equiv f_{\text {hom }}$. The proof of the independence on the space variable proceeds as for the $L^{p}$ case. In order to prove that $f \equiv f_{\text {hom }}$ we first observe that, by the convexity of $f$ and Corollary 4.6 it holds

$$
\begin{aligned}
f(z) & =\lim _{\rho \rightarrow 0} \frac{1}{r^{N}} \min \left\{\int_{Q_{r}} f(u) d x,\langle u\rangle_{Q_{r}} \in \bar{B}(z, \rho)\right\} \\
& =\lim _{\rho \rightarrow 0} \lim _{n} \frac{1}{r^{N}} \inf \left\{F_{\varepsilon_{n}}\left(u, Q_{r}\right),\langle u\rangle_{Q_{r}}^{d, \varepsilon_{n}} \in \bar{B}(z, \rho)\right\} .
\end{aligned}
$$

Analogously to the $L^{p}$ case we scale the problem as follows. Setting $h_{n}=\left[\frac{r}{\varepsilon_{n}}\right]+1$, through the change of variable

$$
\beta=\frac{\alpha}{\varepsilon}, \quad v(\beta)=u(\varepsilon \beta),
$$

equality (5.44) becomes

$f(z)=\lim _{\rho \rightarrow 0} \lim _{n \rightarrow+\infty} \frac{1}{h_{n}^{N}} \inf \left\{\sum_{\xi \in \mathbb{Z}^{N}} \sum_{\beta \in R_{1}^{\xi}\left(Q_{h_{n}}\right)} f^{\xi}(\beta, v(\beta), v(\beta+\xi)),\langle v\rangle_{Q_{h_{n}}}^{d} \in \bar{B}(z, \rho)\right\}$.

The conclusion follows by proving the existence of the first limit in (5.43) for any $\rho>0$. This can be done by repeating the same construction used for the $L^{p}$ case.

\section{Ferromagnetic-antiferromagnetic systems: exis- tence of the bulk limit}

In this section, we recall the model dealt with in [19] and prove that it can be recast in the present setting, the family of energies that arises satisfying the hypotheses of Theorem 5.3. 
Given an integer $M$, let $\Lambda_{M}$ denote $\left[-M, M\left[{ }^{d} \cap \mathbb{Z}^{N}\right.\right.$. The energy of a $\Lambda_{M^{-}}$ periodic configuration $\underline{\sigma}: \Lambda_{M} \rightarrow\{-1,1\}$ is given by

$$
H_{M}(\underline{\sigma})=-J \sum_{k=1}^{N} \sum_{i \in \Lambda_{M}} \sigma_{i} \sigma_{i+e_{k}}+\sum_{i, j \in \Lambda_{M}, i \neq j} \sigma_{i} J_{p}(j-i) \sigma_{j},
$$

where $J>0$ (and if $i+e_{k} \notin \Lambda_{M}$ we assume $\sigma_{i+e_{k}}=\sigma_{i-2 M e_{k}}$ ), and $J_{p}$ is defined, for $p>1$, by

$$
J_{p}(j-i)=\sum_{k \in \mathbb{Z}^{N}} \frac{1}{|i-j+2 k M|^{p}} .
$$

The first term of (6.45) models the ferromagnetic interactions between nearest neighbors (with periodic conditions, which means that the whole space $\mathbb{Z}^{N}$ is covered with the periodic replication of $\Lambda_{M}$ ) and is called the 'exchange energy'. The second term models the antiferromagnetic interactions at long range (also with periodic boundary conditions). It is the 'dipolar energy'. Heuristically, short range interactions prefer uniform states (either of +1 or -1 ), and long range interactions favor alternating states $(+1,-1)$.

The problem of the variational convergence of $\frac{H_{M}(\sigma)}{M^{N}}$ as $M \rightarrow+\infty$ can be equivalently studied on a fixed domain $\Lambda=[-1,1)^{N}$. To this end we set $\varepsilon=\frac{1}{M}$ and, for any $\underline{\sigma}: \Lambda_{M} \rightarrow\{-1,1\}, u(\alpha):=\underline{\sigma}\left(\frac{\alpha}{\varepsilon}\right)$ for all $\alpha \in \varepsilon \mathbb{Z}^{N} \cap \Lambda$. Then, up to lower order terms, we can rewrite $\frac{H_{M}(\bar{\sigma})}{M^{N}}$ as follows:

$$
F_{\varepsilon}(u)=F_{\varepsilon}^{1}(u)+F_{\varepsilon}^{2}(u),
$$

where

$F_{\varepsilon}^{1}(u)=-J \sum_{k=1}^{N} \sum_{\alpha \in R_{\varepsilon}^{e_{k}}(\Lambda)} \varepsilon^{d} u(\alpha) u\left(\alpha+\varepsilon e_{k}\right)+\sum_{\alpha_{1}, \alpha_{2} \in \varepsilon \mathbb{Z}^{N} \cap \Lambda: \alpha_{1} \neq \alpha_{2}} \varepsilon^{N} \varepsilon^{p} \frac{u\left(\alpha_{1}\right) u\left(\alpha_{2}\right)}{\left|\alpha_{1}-\alpha_{2}\right|^{p}}$,

and

$$
\begin{aligned}
& F_{\varepsilon}^{2}(u)=\sum_{\alpha_{1}, \alpha_{2} \in \varepsilon \mathbb{Z}^{N} \cap \Lambda: \alpha_{1} \neq \alpha_{2}} \varepsilon^{N} \sum_{k \in \mathbb{Z}^{N} \backslash\{0\}} \varepsilon^{p} \frac{u\left(\alpha_{1}\right) u\left(\alpha_{2}\right)}{\left|\alpha_{1}-\alpha_{2}+2 k\right|^{p}} \\
& =\sum_{\alpha_{1}, \alpha_{2} \in \varepsilon \mathbb{Z}^{N} \cap \Lambda: \alpha_{1} \neq \alpha_{2}} \varepsilon^{N}\left(f_{1}^{\varepsilon}\left(\alpha_{1}-\alpha_{2}, u\left(\alpha_{1}\right), u\left(\alpha_{2}\right)\right)\right. \\
& \left.+f_{2}^{\varepsilon}\left(\alpha_{1}-\alpha_{2}, u\left(\alpha_{1}\right), u\left(\alpha_{2}\right)\right)\right)
\end{aligned}
$$

where

$$
\begin{aligned}
& f_{1}^{\varepsilon}(z, u, v)=\sum_{k \in \mathbb{Z}^{N},|k|>\sqrt{N}+1} \frac{\varepsilon^{p} u v}{|z+2 k|^{p}}, \\
& f_{2}^{\varepsilon}(z, u, v)=\sum_{0<|k| \leq \sqrt{N}+1} \frac{\varepsilon^{p} u v}{|z+2 k|^{p}} .
\end{aligned}
$$


Let us prove that for $p>N$,

$$
\lim _{\varepsilon \rightarrow 0} F_{\varepsilon}^{2}(u)=0
$$

uniformly with respect to $u$. Once (6.46) is proved, we have

$$
\Gamma-\lim _{\varepsilon \rightarrow 0} F_{\varepsilon}(u)=\Gamma-\lim _{\varepsilon \rightarrow 0} F_{\varepsilon}^{1}(u) .
$$

In addition, $F_{\varepsilon}^{1}(u)$ can be rewritten as

$$
F_{\varepsilon}^{1}(u)=-J \sum_{k=1}^{N} \sum_{\alpha \in R_{\varepsilon}^{e_{k}}(\Lambda)} \varepsilon^{N} u(\alpha) u\left(\alpha+\varepsilon e_{k}\right)+\sum_{\xi \in \mathbb{Z}^{N}} \sum_{\alpha \in R_{\varepsilon}^{\xi}(\Lambda)} \varepsilon^{N} \frac{u(\alpha) u(\alpha+\varepsilon \xi)}{|\xi|^{p}}
$$

and turns out to satisfy the hypotheses of Theorem 5.3 for $p>N$. This implies the integral representation property of its $\Gamma$-limit.

To prove (6.46) we first estimate the term in the energy with $f_{1}^{\varepsilon}$. Since $\mid \alpha_{1}-$ $\alpha_{2} \mid<2 \sqrt{N}$ and $k>\sqrt{N}+1$, by applying the triangular inequality, we have that

$$
\left|\alpha_{1}-\alpha_{2}+2 k\right|^{p} \geq|| 2 k|-| \alpha_{1}-\left.\alpha_{2}\right|^{p} \geq|2 k|^{p}\left|1-\frac{\left|\alpha_{1}-\alpha_{2}\right|}{|2 k|}\right|^{p} \geq C|2 k|^{p} .
$$

Thus

$$
\left|f_{1}^{\varepsilon}(z, u, v)\right| \leq C \varepsilon^{p} \sum_{k \in \mathbb{Z}^{N},|k| \neq 0} \frac{1}{|2 k|^{p}} \leq C \varepsilon^{p}
$$

for $p>N$, and

$$
\begin{aligned}
& \sum_{\substack{\alpha_{1}, \alpha_{2} \in \varepsilon \mathbb{Z}^{N} \cap \Lambda: \alpha_{1} \neq \alpha_{2} \\
\leq}} \varepsilon^{N} \sum_{\substack{\alpha_{1} \\
\alpha_{1}, \alpha_{2} \in \varepsilon \mathbb{Z}^{N} \cap \Lambda: \alpha_{1} \neq \alpha_{2} \\
\leq}} \varepsilon^{p} \sum_{k \in \varepsilon^{N+p} \varepsilon^{-2 N}=C \varepsilon^{p-N} .|k| \neq 0} \frac{1}{|2 k|^{p}} \\
&
\end{aligned}
$$

To estimate the term with $f_{2}^{\varepsilon}$ one has to be more precise. Noting that $\mid \alpha_{1}-\alpha_{2}+$ $2 k \mid \geq \varepsilon$ we collect the interactions according to a logarithmic scale in $\varepsilon$ as follows:

$$
\begin{aligned}
\sum_{\alpha_{1}, \alpha_{2} \in \varepsilon \mathbb{Z}^{N} \cap \Lambda:} \varepsilon^{N} \mid f_{1}^{\varepsilon} \neq \alpha_{2} \\
\leq \varepsilon^{N+p} \sum_{\substack{0<|k|<\sqrt{N}+1\\
}} \sum_{i=0}^{M-1} \sum_{\substack{\alpha_{1}, \alpha_{2} \in I_{i}\\
}} \frac{1}{\left|\varepsilon_{1}-\alpha_{2}+2 k\right|^{p}} \\
\sum_{0<|k|<\sqrt{N}+1} \sum_{\left|\alpha_{1}-\alpha_{2}+2 k\right| \geq 1} \frac{1}{\left|\alpha_{1}-\alpha_{2}+2 k\right|^{p}}
\end{aligned}
$$


where, for $i \in\{0,1, \ldots, M-1\}$, we have set

$$
I_{i}=\left\{\left(\alpha_{1}, \alpha_{2}\right) \in\left(\varepsilon \mathbb{Z}^{N} \cap \Lambda\right)^{2}: \varepsilon^{\frac{i+1}{M}} \leq\left|\alpha_{1}-\alpha_{2}+2 k\right|<\varepsilon^{\frac{i}{M}}\right\} .
$$

Since $I_{i} \subset \tilde{I}_{i}:=\left\{\left(\alpha_{1}, \alpha_{2}\right) \in\left(\varepsilon \mathbb{Z}^{N} \cap \Lambda\right)^{2}:\left|\alpha_{1}-\alpha_{2}+2 k\right|<\varepsilon^{\frac{i}{M}}\right\}$, we have that

$$
\#\left(I_{i}\right) \leq \#\left(\tilde{I}_{i}\right) \leq C \varepsilon^{\frac{(N+1) i}{M}} \varepsilon^{-2 N} .
$$

Set, for $\eta>0 I^{\eta}:=\left\{\alpha_{1}, \alpha_{2} \in \varepsilon \mathbb{Z}^{N} \cap \Lambda:\left|\alpha_{1}-\alpha_{2}+2 k\right| \leq \eta\right\}$. One can show $\#\left(I^{\eta}\right) \leq C\left(\frac{\eta}{\varepsilon^{N}}\right)\left(\frac{\eta^{N}}{\varepsilon^{N}}\right)$. Since

$$
\varepsilon^{N+p} \sum_{0<|k|<\sqrt{N}+1} \sum_{\left|\alpha_{1}-\alpha_{2}+2 k\right| \geq 1} \frac{1}{\left|\alpha_{1}-\alpha_{2}+2 k\right|^{p}} \leq C \varepsilon^{p-N}
$$

we only need to estimate the first term in the right hand side of (6.47) to conclude. Using (6.48), we have

$$
\begin{gathered}
\varepsilon^{N+p} \sum_{0<|k|<\sqrt{N+1}} \sum_{i=0}^{M-1} \sum_{\alpha_{1}, \alpha_{2} \in I_{i}} \frac{1}{\left|\alpha_{1}-\alpha_{2}+2 k\right|^{p}} \leq C \varepsilon^{N+p} \sum_{i=0}^{M-1} \frac{\#\left(I_{i}\right)}{\varepsilon^{\frac{p(i+1)}{M}}} \\
\leq C \varepsilon^{p-N} \sum_{i=0}^{M-1} \varepsilon^{\frac{(N+1-p) i}{M}} \varepsilon^{-\frac{p}{M}} \\
=C \varepsilon^{p-N-\frac{p}{M}} \sum_{i=0}^{M-1}\left(\varepsilon^{\frac{(N+1-p)}{M}}\right)^{i}=: L(\varepsilon, M) .
\end{gathered}
$$

If $p=N+1$ then

$$
L(\varepsilon, M) \leq C M \varepsilon^{1-\frac{N+1}{M}}
$$

which converges to zero as $\varepsilon \rightarrow 0$ provided $M$ is chosen large enough. If $p \neq N+1$, let $q=\varepsilon^{\frac{N+1-p}{M}}$. As $\sum_{i=0}^{M-1} q^{i}=\frac{1-q^{M}}{1-q}$, there holds

$$
L(\varepsilon, M) \leq C \varepsilon^{p-N-\frac{p}{M}}\left(\frac{1-\varepsilon^{N+1-p}}{1-\varepsilon^{N+1-p M}}\right) .
$$

It is then easy to verify that the last term converges to zero as $\varepsilon \rightarrow 0$ for $M$ large enough.

\section{Non-pairwise-interaction energies}

In this section we deal with more general discrete systems driven by non pairwiseinteraction energies. Given $k \in \mathbb{N}$, the energy for such discrete systems is defined, for any $u \in C_{\varepsilon}\left(\Omega, \mathbb{R}^{m}\right)$, by

$$
F_{\varepsilon}(u)=\sum_{j=1}^{k} \sum_{\bar{\xi}_{j} \in \mathbb{Z}^{j N}} \sum_{\alpha \in R_{\varepsilon, j}^{\bar{\xi}_{j}}(\Omega)} \varepsilon^{N} f_{\varepsilon, j}^{\bar{\xi}_{j}}\left(\alpha, u(\alpha), u\left(\alpha+\varepsilon \xi_{1}\right), \ldots, u\left(\alpha+\varepsilon \xi_{j}\right)\right)
$$


where $\bar{\xi}_{j}=\left(\xi_{1}, \xi_{2}, \ldots, \xi_{j}\right) \in \mathbb{Z}^{j N}$ and

$$
R_{\varepsilon, j}^{\bar{\xi}_{j}}(\Omega)=\left\{\alpha \in \mathbb{Z}_{\varepsilon}(\Omega): \alpha, \alpha+\varepsilon \xi_{1}, \ldots, \alpha+\varepsilon \xi_{j} \in \mathbb{Z}_{\varepsilon}(\Omega)\right\} .
$$

It may be checked that the arguments we have used so far in the case of pairwiseinteracting discrete systems can be exploited in order to treat more general systems driven by energies of the form (7.50) provided that we modify assumptions (H1)(H6) by substituting in each formula $\xi$ by $\bar{\xi}_{j}$ and $|\xi|$ by $\left\|\bar{\xi}_{j}\right\|_{\infty}:=\max _{i \in\{1, \ldots, j\}}\left|\xi_{i}\right|$. More precisely, in the $L^{p}$ case, conditions (H1)-(H3) are replaced by:

(H11) For all $j \in\{1,2, \ldots, k\}, \bar{\xi}_{j} \in \mathbb{Z}^{j N}, \alpha \in \mathbb{Z}_{\varepsilon}(\Omega)$ and $\varepsilon>0$, there exist $c_{\varepsilon, \alpha}^{\bar{\xi}_{j}} \geq 0$ and $d_{\varepsilon}^{\bar{\xi}_{j}} \in C_{\varepsilon}(\Omega, \mathbb{R}), d_{\varepsilon}^{\bar{\xi}_{j}}(\alpha) \geq 0$ such that

$$
\begin{aligned}
& f_{\varepsilon, j}^{\bar{\xi}_{j}}\left(\alpha, u_{1}, u_{2}, \ldots, u_{j}\right) \geq c_{\varepsilon, \alpha}^{\bar{\xi}_{j}}\left(\sum_{i=1}^{j}\left|u_{i}\right|^{p}-d_{\varepsilon}^{\bar{\xi}_{j}}(\alpha)\right) \quad \text { for all }\left(u_{1}, u_{2}, \ldots, u_{j}\right) \in \mathbb{R}^{j m}, \\
& \lim _{R \rightarrow \infty} \liminf _{\varepsilon \rightarrow 0} \inf _{\alpha \in \mathbb{Z}_{\varepsilon}(\Omega)} \sum_{j=1}^{k} \sum_{\substack{\bar{\xi}_{j} \in \mathbb{Z}^{j N}: \\
\left\|\bar{\xi}_{j}\right\|_{\infty} \leq R}} \bar{c}_{\varepsilon, \alpha} \geq c>0
\end{aligned}
$$

and the function $d_{\varepsilon} \in C_{\varepsilon}(\Omega, \mathbb{R})$ defined by $d_{\varepsilon}(\alpha)=\sum_{j=1}^{k} \sum_{\bar{\xi}_{j} \in \mathbb{Z}^{j N}} d_{\varepsilon}^{\bar{\xi}_{j}}(\alpha)$ weakly converges to $d$ in $L^{1}(\Omega)$.

(H12) For all $j \in\{1,2, \ldots, k\}, \bar{\xi}_{j} \in \mathbb{Z}^{j N}, \alpha \in \mathbb{Z}_{\varepsilon}(\Omega)$ and $\varepsilon>0$ there exist $C_{\varepsilon, \alpha}^{\bar{\xi}_{j}} \geq 0$ and $D_{\varepsilon}^{\bar{\xi}_{j}} \in C_{\varepsilon}(\Omega, \mathbb{R}), D_{\varepsilon}^{\bar{\xi}_{j}}(\alpha) \geq 0$ such that

$$
\begin{aligned}
& f_{\varepsilon, j}^{\bar{\xi}_{j}}\left(\alpha, u_{1}, u_{2}, \ldots, u_{j}\right) \leq C_{\varepsilon, \alpha}^{\bar{\xi}_{j}}\left(\sum_{i=1}^{j}\left|u_{i}\right|^{p}+D_{\varepsilon}^{\bar{\xi}_{j}}(\alpha)\right) \text { for all }\left(u_{1}, u_{2}, \ldots, u_{j}\right) \in \mathbb{R}^{j m}, \\
& \limsup _{\varepsilon \rightarrow 0} \sup _{\alpha \in \mathbb{Z}_{\varepsilon}(\Omega)} \sum_{j=1}^{k} \sum_{\substack{\bar{\xi}_{j} \in \mathbb{Z}^{j N}: \\
\left\|\bar{\xi}_{j}\right\|_{\infty} \leq R}} C_{\varepsilon, \alpha}^{\bar{\xi}_{j}} \leq C<\infty
\end{aligned}
$$

and the function $D_{\varepsilon} \in C_{\varepsilon}(\Omega, \mathbb{R})$ defined by $D_{\varepsilon}(\alpha)=\sum_{j=1}^{k} \sum_{\bar{\xi}_{j} \in \mathbb{Z}^{j N}} D_{\varepsilon}^{\bar{\xi}_{j}}(\alpha)$ weakly converges to $D$ in $L^{1}(\Omega)$. 
(H13) For all $\delta>0$, there exists $M_{\delta}>0$ such that

$$
\limsup _{\varepsilon \rightarrow 0} \sup _{\alpha \in \mathbb{Z}_{\varepsilon}(\Omega)} \sum_{j=1}^{k} \sum_{\substack{\bar{\xi}_{j} \in \mathbb{Z}^{j N}: \\\left\|\bar{\xi}_{j}\right\|_{\infty} \geq M_{\delta}}} C_{\varepsilon, \alpha}^{\bar{\xi}_{j}} \leq \delta .
$$

Under hypotheses (H11)-(H13) the analogues of Theorems 3.1 and 3.13 hold.

In the $L^{\infty}$ case hypotheses (H4)-(H6) are replaced by:

(H14) For all $j \in\{1,2, \ldots, k\}, \bar{\xi}_{j} \in \mathbb{Z}^{j N}, \alpha \in \mathbb{Z}_{\varepsilon}(\Omega)$ and $\varepsilon>0$,

$$
f_{\varepsilon, j}^{\bar{\xi}_{j}}\left(\alpha, u_{1}, u_{2}, \ldots, u_{j}\right)=+\infty \quad \text { if }\left(u_{1}, u_{2}, \ldots, u_{j}\right) \notin K^{j} .
$$

(H15) For all $j \in\{1,2, \ldots, k\}, \bar{\xi}_{j} \in \mathbb{Z}^{j N}, \alpha \in \mathbb{Z}_{\varepsilon}(\Omega)$ and $\varepsilon>0$, there exists $C_{\varepsilon, \alpha}^{\bar{\xi}_{j}} \geq 0$ such that

$$
\begin{aligned}
& \left|f_{\varepsilon}^{\bar{\xi}_{j}}\left(\alpha, u_{1}, u_{2}, \ldots, u_{j}\right)\right| \leq C_{\varepsilon, \alpha}^{\bar{\xi}_{j}} \quad \text { for all }\left(u_{1}, u_{2}, \ldots, u_{j}\right) \in K^{j}, \\
& \limsup _{\varepsilon \rightarrow 0} \sup _{\alpha \in \mathbb{Z}_{\varepsilon}(\Omega)} \sum_{j=1}^{k} \sum_{\bar{\xi}_{j} \in \mathbb{Z}^{j N}} C_{\varepsilon, \alpha}^{\bar{\xi}_{j}}<\infty .
\end{aligned}
$$

(H16) For all $j \in\{1,2, \ldots, k\}, \bar{\xi}_{j} \in \mathbb{Z}^{j N}, \alpha \in \mathbb{Z}_{\varepsilon}(\Omega), \varepsilon>0$ and $\delta>0$, there exists $M_{\delta}>0$ such that

$$
\limsup _{\varepsilon \rightarrow 0} \sup _{\alpha \in \mathbb{Z}_{\varepsilon}(\Omega)} \sum_{j=1}^{k} \sum_{\substack{\bar{\xi}_{j} \in \mathbb{Z}^{j N}: \\\|\bar{\xi}\|_{\infty} \geq M_{\delta}}} C_{\varepsilon, \alpha}^{\bar{\xi}_{j}} \leq \delta .
$$

Under hypotheses (H14)-(H16) the analogue of Theorems 3.3 and 3.14 hold.

If in addition to the previous assumptions we consider periodicity hypotheses on $f_{\varepsilon, j} \bar{\xi}_{j}$, the homogenization theory developed in Sections 5.1 and 5.2 can be extended to the present case.

\section{Remark 7.1 (More general lattices)}

The present result can be extended to the case of energies of the type (7.50), but defined on more general lattices. In particular, given $\left\{\eta_{1}, \eta_{2}, \ldots, \eta_{N}\right\}$ a base in $\mathbb{R}^{N}$, the case of a discrete spin system on the simple lattice $\tilde{\mathbb{Z}}:=\bigoplus_{i=1}^{N} \eta_{i} \mathbb{Z}$ can be easily addressed by following the same strategy we have used to treat the $\mathbb{Z}^{N}$ case. Note that for the simple lattice $\tilde{\mathbb{Z}}$, one may identify any $u: \varepsilon \tilde{\mathbb{Z}} \cap \Omega \rightarrow \mathbb{R}^{m}$ with the piecewise constant function $u$ belonging to the set

$$
\tilde{C}_{\varepsilon}\left(\Omega, \mathbb{R}^{m}\right):=\left\{u: \mathbb{R}^{N} \rightarrow \mathbb{R}^{m}: u(x)=u(\alpha) \forall x \in \alpha+\varepsilon \tilde{Q}, \alpha \in \varepsilon \tilde{\mathbb{Z}}\right\},
$$

where $\tilde{Q}:=\left\{x \in \mathbb{R}^{N}: x=\sum_{i=1}^{N} \lambda_{i} \eta_{i}, \lambda_{i} \in[0,1)\right\}$. 


\subsection{Multiple-spin exchange energies}

An important class of non pairwise-interacting discrete systems to which all the previous result apply, is provided by Heisenberg spin systems driven by energies containing multiple-spin exchange terms, namely energies that, for any $u \in$ $C_{\varepsilon}(\Omega, K)$, are of the form

$$
F_{\varepsilon}(u)=\sum_{j=2}^{k} J_{j} \sum_{I^{j}} \varepsilon^{N} u\left(\alpha_{1}\right) u\left(\alpha_{2}\right) \ldots u\left(\alpha_{j}\right),
$$

where $K \in \mathbb{R}^{m}$ is a bounded set, $k \geq 3$ and for all $j \in\{1, \ldots, k\}$, the constant $J_{j}$ is also known as the exchange constant of the $j$-body nearest-neighbors interaction. Here $I^{j}$ denotes a set of $j$-ples of points of the lattice subject to some constraints which further specify the model.

In order to state precisely the constraints for some cases of interest, let us first introduce some additional definitions. Using the notation of in Remark 7.1, we denote by $\tilde{\mathbb{Z}}$ a $N$-dimensional simple lattice and we set $\tilde{\mathbb{Z}}_{\varepsilon}(\Omega)=\varepsilon \tilde{\mathbb{Z}} \cap \Omega$. Given $k \geq 3$ and a $k$-ple $\left(\alpha_{1}, \alpha_{2}, \ldots, \alpha_{k}\right) \in\left(\tilde{\mathbb{Z}}_{\varepsilon}(\Omega)\right)^{k}$ with $\alpha_{i} \neq \alpha_{j}$, we say that the $k$-ple is a $k$-body chain of nearest-neighbors (or shortly a $k$-chain) if, for all $j \in\{2,3, \ldots, k-1\}$, each $\alpha_{j}$ is a nearest neighbour for $\alpha_{j-1}$ and $\alpha_{j+1}$ (see Figure 7.1). We say that a $k$-chain is a $k$-cycle of nearest neighbors (or shortly a $k$-cycle) if, $\alpha_{1}$ is a nearest neighbour for $\alpha_{k}$ (see Figure 7.1). Given a set $V \subset \Omega$, we say that a $k$-chain $\left(\alpha_{1}, \alpha_{2}, \ldots, \alpha_{k}\right)$ is contained in $V$ if $\left\{\alpha_{1}, \alpha_{2}, \ldots, \alpha_{k}\right\} \subset V$.

Discrete systems driven by energies of the form (7.51) with

$$
I^{k}:=\left\{\left(\alpha_{1}, \alpha_{2}, \ldots, \alpha_{k}\right) \in\left(\tilde{\mathbb{Z}}_{\varepsilon}(\Omega)\right)^{k}:\left(\alpha_{1}, \alpha_{2}, \ldots, \alpha_{k}\right) \text { is a } k \text {-chain }\right\},
$$

or

$$
I^{k}:=\left\{\left(\alpha_{1}, \alpha_{2}, \ldots, \alpha_{k}\right) \in\left(\tilde{\mathbb{Z}}_{\varepsilon}(\Omega)\right)^{k}:\left(\alpha_{1}, \alpha_{2}, \ldots, \alpha_{k}\right) \text { is a } k \text {-cycle }\right\},
$$

have been extensively studied for different values of the exchange constants both from the analytical and the computational points of view (see e.g. [6], [20], [25]). Even if in general it is not easy to guess the explicit formula for the bulk limit, let us point out that the homogenization result holds for both cases and provides the existence of a local limit energy of integral type and an implicit asymptotic formula for its energy density.

We conclude this section with an example of a two-dimensional ferromagnetic model with 3-spin exchange energy for which it is possible to explicitly write the limit energy.

Example 7.2 Let us consider $\Omega \subset \mathbb{R}^{2}$ and $K=\{-1,1\}$. In what follows we consider a spin system driven by an energy of the form (7.51) both on a triangular lattice and on a square lattice. After providing an explicit formula for the limit energy density in both cases, we discuss its dependence upon the geometry of the lattice. 

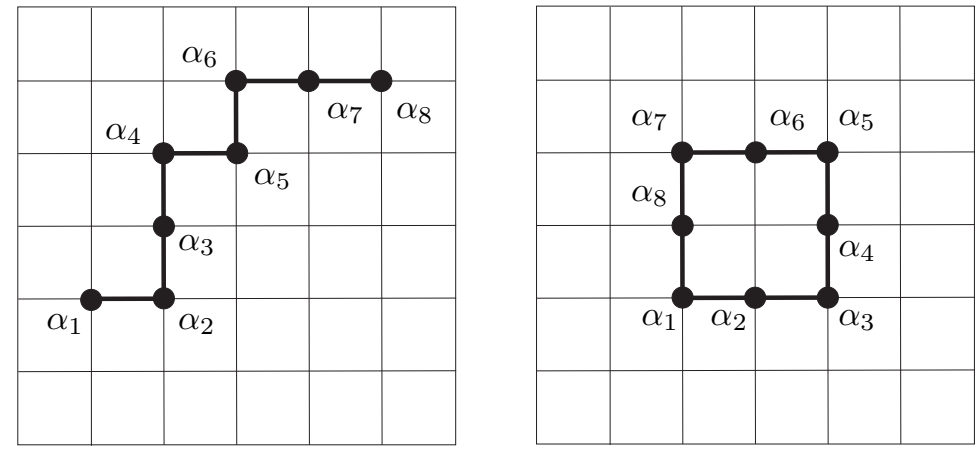

Figure 1: an example of 8-chain (left) and 8-cycle (right)

Let us consider a regular triangular lattice, that is $\tilde{\mathbb{Z}}=\eta_{1} \mathbb{Z} \oplus \eta_{2} \mathbb{Z}$ where $\eta_{1}=(1,0)$ and $\eta_{2}=\left(\frac{1}{2}, \frac{\sqrt{3}}{2}\right)$. By analogy with the $\mathbb{Z}^{2}$ lattice, where a cell is the minimal square with vertices in $\mathbb{Z}^{2}$, in the triangular case we denote by 'cell' the minimal equilateral triangle with vertices in $\tilde{\mathbb{Z}}$. Then, for $k=3, J_{2}=0$ (the case $J_{2} \neq 0$ can be dealt with similarly) and $J_{3}=-1$ we consider the energy

$$
F_{\varepsilon}(u)=-\sum_{\left(\alpha_{1}, \alpha_{2}, \alpha_{3}\right) \in I} \varepsilon^{2} u\left(\alpha_{1}\right) u\left(\alpha_{2}\right) u\left(\alpha_{3}\right) .
$$

where $I$ is the set of all 3-chains contained in a cell of the lattice.

Case (i): triangular lattice. The energies in (7.52) are of the type (7.50) with $N=2, \mathbb{Z}$ instead of $\mathbb{Z}^{2}$ and

$$
f_{\varepsilon}^{\bar{\xi}}\left(\alpha, u_{1}, u_{2}, u_{3}\right)= \begin{cases}-u_{1} u_{2} u_{3} & \text { if } \bar{\xi}= \pm\left(\eta_{1}, \eta_{2}\right) \\ 0 & \text { otherwise }\end{cases}
$$

To find the explicit form of the $\Gamma$-limit we may use an approach similar to the one exploited in [2]. The energy in (7.52) can be rewritten as parameterized by the centers of the cells of $\tilde{\mathbb{Z}}$; that is, by the points $\beta=\frac{\alpha_{1}+\alpha_{2}+\alpha_{3}}{3}$, with $\alpha_{1}, \alpha_{2}, \alpha_{3} \in \tilde{\mathbb{Z}}$ being the vertices of a cell. Then

$$
F_{\varepsilon}(u)=\sum_{\beta} \varepsilon^{2} g(v(\beta))
$$

with $v(\beta)=\sum_{i=1}^{3} u\left(\alpha_{i}\right)$. Here $v \in\left\{-1,-\frac{1}{3}, \frac{1}{3}, 1\right\}$ and $g:\left\{-1,-\frac{1}{3}, \frac{1}{3}, 1\right\} \rightarrow \mathbb{R}$ is given by

$$
g(z)= \begin{cases}-1 & \text { if } z \in\left\{-\frac{1}{3}, 1\right\} \\ +1 & \text { if } z \in\left\{-1, \frac{1}{3}\right\}\end{cases}
$$


Observe that this change of variables allows us to regard the multiple-exchange spin-type energy in (7.52) as an energy of a non-interacting spin system. Moreover note that if $u_{\varepsilon} \rightarrow^{*} u$ in $L^{\infty}(\Omega)$, then $v_{\varepsilon}$ (extended to $\mathbb{R}^{N}$ with constant value $v_{\varepsilon}(\beta)$ in the triangle centered in $\beta$ ) still converges to $u$ in the $w^{*}$-topology of $L^{\infty}(\Omega)$. This argument shows that the $\Gamma$-limit of $F_{\varepsilon}$ is given by a convexification procedure. Indeed it can be proved that

$$
\Gamma\left(w^{*}-L^{\infty}\right)-\lim F_{\varepsilon}(u)=\int_{\Omega} \bar{g}^{* *}(u(x)) d x,
$$

where $\bar{g}: \mathbb{R} \rightarrow \mathbb{R} \cup+\infty$ is given by

$$
\bar{g}(z)= \begin{cases}g(z) & \text { if } z \in\left\{-1,-\frac{1}{3}, \frac{1}{3}, 1\right\} \\ +\infty & \text { otherwise }\end{cases}
$$

and $\bar{g}^{* *}$ stands for the convex envelope of $\bar{g}$; i.e.

$$
\bar{g}^{* *}(z)= \begin{cases}-3 z-2 & \text { if }-1 \leq z \leq-\frac{1}{3} \\ -1 & \text { if }-\frac{1}{3} \leq z \leq 1 \\ +\infty & \text { otherwise }\end{cases}
$$

Case (ii): square lattice In this case the energies in (7.52) are of the type (7.50) with $N=2$ and

$$
f_{\varepsilon}^{\bar{\xi}}\left(\alpha, u_{1}, u_{2}, u_{3}\right)= \begin{cases}-u_{1} u_{2} u_{3} & \text { if } \bar{\xi} \in\left\{ \pm\left(e_{1}, e_{2}\right), \pm\left(e_{1},-e_{2}\right)\right\} \\ 0 & \text { otherwise. }\end{cases}
$$

Arguing as before, we may rewrite the energy as parameterized by the centers of the cells of the lattice $\mathbb{Z}^{2}$; that is, by the points $\beta=\frac{\alpha_{1}+\alpha_{2}+\alpha_{3}+\alpha_{4}}{4}$, with $\alpha_{1}, \alpha_{2}, \alpha_{3}, \alpha_{4} \in$ $\mathbb{Z}^{2}$ being the vertices of a cell. Then

$$
F_{\varepsilon}(u)=\sum_{\beta} \varepsilon^{2} h(v(\beta))
$$

with $v(\beta)=\sum_{i=1}^{4} u\left(\alpha_{i}\right)$. Note that $v \in\left\{-1,-\frac{1}{2}, 0, \frac{1}{2}, 1\right\}$ and that $h:\left\{-1,-\frac{1}{2}, 0, \frac{1}{2}, 1\right\} \rightarrow$ $\mathbb{R}$ is given by

$$
h(z)= \begin{cases}4 & \text { if } z=-1 \\ -2 & \text { if } z=-\frac{1}{2} \\ 0 & \text { if } z=0 \\ 2 & \text { if } z=\frac{1}{2} \\ -4 & \text { if } z=1\end{cases}
$$

As in the previous case, if $u_{\varepsilon} \rightarrow^{*} u$ in $L^{\infty}(\Omega)$, after extending $v_{\varepsilon}$ to a piecewiseconstant function on the cells of the lattice $\mathbb{Z}^{2}$, we have $v_{\varepsilon} \rightarrow^{*} u$ in $L^{\infty}(\Omega)$. In this case it can be proved that

$$
\Gamma\left(w^{*}-L^{\infty}\right)-\lim F_{\varepsilon}(u)=\int_{\Omega} \bar{h}^{* *}(u(x)) d x,
$$



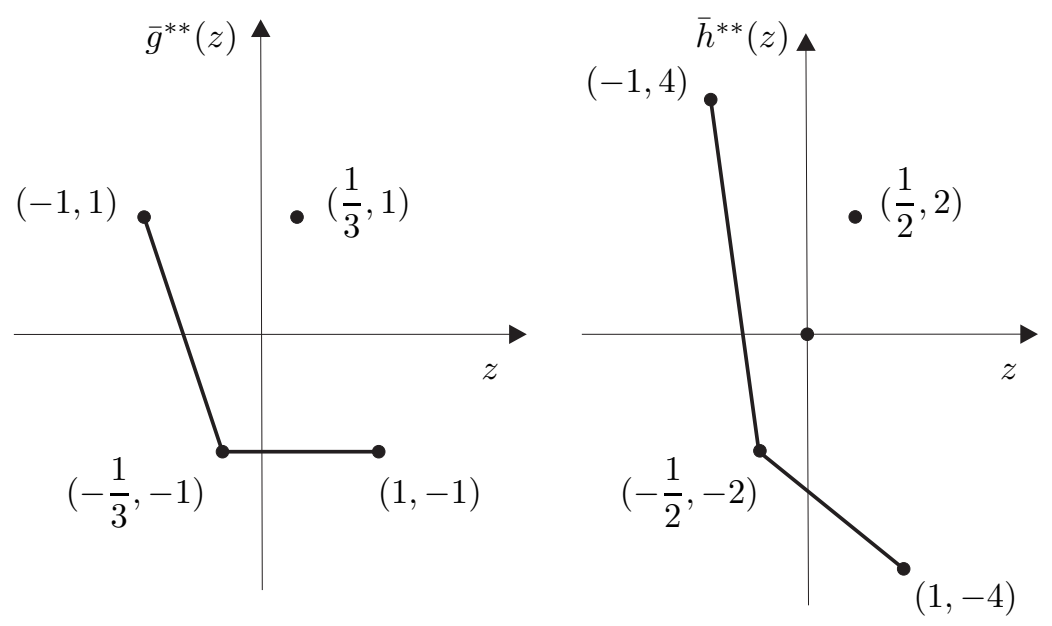

Figure 2: the energy densities in the triangular (left) and square (right) cases in example 7.2

where $\bar{h}: \mathbb{R} \rightarrow \mathbb{R} \cup+\infty$ is given by

$$
\bar{h}(z)= \begin{cases}h(z) & \text { if } z \in\left\{-1,-\frac{1}{2}, 0, \frac{1}{2}, 1\right\} \\ +\infty & \text { otherwise }\end{cases}
$$

and $\bar{h}^{* *}$ is given by

$$
\bar{h}^{* *}(z)=\left\{\begin{array}{lc}
-12 z-8 & \text { if }-1 \leq z \leq-\frac{1}{2} \\
-\frac{4}{3} z-\frac{8}{3} & \text { if }-\frac{1}{2} \leq z \leq 1 \\
+\infty & \text { otherwise }
\end{array}\right.
$$

We remark that some features of the energy density obtained in the two cases are peculiar of the geometric frustration of the system (see [18] and [23] for an introduction to the subject). For the type of energies considered here, the triangular case is an example of non-frustrated system, while the square case is a frustrated spin system (the geometric frustration can be seen in the fact that the triple of values $(-1,-1,1)$ minimizes the energy density but cannot be repeated on the square lattice in order to be minimal on each cell of the lattice). The frustration is responsible for the non-degeneracy of the limit energy (see figure 7.2). This implies that no phase-transition phenomenon occurs at the scale $\varepsilon$ for the square lattice. Whereas, for the triangular lattice, the limit energy density $\bar{g}^{* *}$ has multiple minima, which allows for phase transitions at the scale $\varepsilon$. 
Acknowledgements. We thank Andrea Braides and Errico Presutti for interesting discussions related to thermodynamic limits, phase transitions and discrete systems, and acknowledge support from the Marie Curie Research Training Network MRTN-CT-2004-505226 'Multi-scale modelling and characterisation for phase transformations in advanced materials' (MULTIMAT).

\section{References}

[1] R. Alicandro, A. Braides and M. Cicalese. Continuum limits of discrete thin films with superlinear growth densities, preprint Scuola Normale Superiore di Pisa, (2007), (download @ http://cvgmt.sns.it/).

[2] R. Alicandro, A. Braides, and M. Cicalese. Phase and anti-phase boundaries in binary discrete systems: a variational viewpoint. Netw. Heterog. Media., $1: 85-107,2006$.

[3] R. Alicandro and M. Cicalese. A general integral representation result for the continuum limits of discrete energies with superlinear growth. SIAM J. Math. Anal., 36(1):1-37, 2004.

[4] R. Alicandro and M. Cicalese. Variational analysis of the asymptotics of the XY model. Preprint, 2007.

[5] J.M. Ball. A version of the fundamental theorem for Young measures. In PDEs and continuum models of phase transitions (Nice 1988), volume 344 of Lecture Notes in Phys., pages 207-215. Springer, Berlin, 1980.

[6] R.J. Baxter. Exactly solved models in statistical mechanics. Academic Press, Inc., London, 1982.

[7] A. Braides. On local variational limits of discrete systems. Comm. Contemporary Math., 2:285-297, 2000.

[8] A. Braides, Г-convergence for Beginners. Oxford University Press, Oxford, 2002.

[9] A. Braides, G. Dal Maso and A. Garroni, Variational formulation of softening phenomena in fracture mechanics: the one-dimensional case, Arch. Rational Mech. Anal. 146 (1999), 23-58.

[10] A. Braides and A. Defranceschi. Homogenization of Multiple Integrals, volume 12 of Oxford Lecture Series in Mathematics and Its Applications. Oxford University Press, 1998.

[11] A. Braides and M.S. Gelli, Limits of discrete systems with long range interactions, J. Convex Anal. 9 (2002), 363-399. 
[12] A. Braides, A.J. Lew and M. Ortiz. Effective cohesive behavior of layers of interatomic planes. Arch. Ration. Mech. Anal., to appear.

[13] A. Braides, L. Truskinowsky, Asymptotic expansions by $\Gamma$-convergence. Preprint, 2007.

[14] G. Buttazzo. Integral representation theory for some classes of local functions, volume 244 of Pitman Res. Notes Math. Ser. Longman Sci. Tech., 1992.

[15] S. Conti, A. De Simone, S. Müller, and F. Otto. Multiscale modeling of materials - the role of analysis. In Trends in nonlinear analysis, pages $375-$ 408, Berlin, 2003. Springer.

[16] G. Dal Maso. An Introduction to $\Gamma$-Convergence. Birkhäuser Boston, Boston, MA, 1993.

[17] A. De Simone, R.V. Kohn, S. Müller, and F. Otto. Magnetic microstructures - a paradigm of multiscale problems. In ICIAM 99 (Edinburgh), pages 175-190, Oxford, 2000. Oxford Univ. Press.

[18] H.T. Diep and H. Giacomini. Frustration - Exactly solved frustrated models. In Frustrated spin systems, H.T. Diep (eds.), World Scientific, 1-58.

[19] A. Giuliani, J.L. Lebowitz, and E.H. Lieb. Ising models with long-range dipolar and short range ferromagnetic interactions. Phys. Rev. B, 74:064420, 2006.

[20] T. Horiguchi. Ising models with two-spin interactions and three-spin interactions on a square lattice. Phys. A, 136(1):109-123, 1986.

[21] R.V. Kohn. The relaxation of a double-well energy. Contin. Mech. Thermodyn., 3(3):193-236, 1991.

[22] R.V. Kohn. Energy-driven pattern formation. In Proceedings of ICM2006, to appear.

[23] R. Liebman. Statistical mechanics of periodic frustrated Ising systems. Lecture Notes in Physics, vol. 251, Springer-Verlag, Berlin, 1986.

[24] J.L. Lebowitz and E. Presutti. Statistical mechanics of systems of unbounded spins. Com. Math. Phys., 50 and 78:195-218 and 151, 1976 and 1980.

[25] A. Malakis. Two- and three-spin triangular Ising model: variational approximations. J. Stat. Phys., 27(1):1-17, 1982.

[26] S. Müller. Variational models for microstructure and phase transitions. In Calculus of variations and geometric evolution problems (Cetraro, 1996), volume 1713 of Lecture Notes in Math., pages 85-210. Springer, Berlin, 1999. 
[27] M. Ponsiglione, Elastic energy stored in a crystal induced by screw dislocations: from discrete to continuous, to appear on SIAM J. Math. Anal.

[28] D. Ruelle. Statistical mechanics. Rigorous results. World Scientific Publishing Co., Inc., River Edge, NJ; Imperial College Press, London, 1999. Reprint of the 1989 edition.

\section{AUthors' ADDRESSES}

Roberto Alicandro

DAEIMI, Università di Cassino

via Di Biasio 43, 03043 Cassino (FR), Italy

e-mail: alicandr@unicas.it

Marco Cicalese

Dipartimento di Matematica e Applicazioni 'R. Caccioppoli', Università di Napoli via Cintia, 80126 Napoli, Italy

e-mail: cicalese@unina.it

Antoine GLORIA

CERMICS - ENPC

6 et 8 avenue Blaise Pascal Cité Descartes - Champs sur Marne, 77455 Marne la Vallée Cedex 2, France

e-mail: gloria@cermics.enpc.fr 